\title{
The Real Truth About the Unreal Future*
}

\author{
Rachael Briggs and Graeme A. Forbes
}

April 5, 2011

Recent times have been very much focussed on the future. The election of Barack Obama in America was accompanied by a wave of optimism. The Global Financial Crisis and the challenge of Climate Change cause many to descend into pessimism. It is not whether our glass is half-empty or half-full that we worry about, but whether it will be empty or full. As philosophers, naturally, we want to illuminate such concerns, so that we might understand them better and subject them to rational scrutiny.

According to the Growing-Block view of time, most famously put forward by C.D. Broad [1923], the flow of time consists in events coming into existence, so that past things and events are real, while future things and events are not. While the Growing-Block view is often considered intuitively appealing, some are concerned it has little to say about the future it denies the existence of. We show how Growing-Block theorists can assign meaningful, mind-independent truth conditions to sentences about the future.

\section{Introducing the Growing Block Theory}

Asymmetries between past and future abound. The past, many of us think, is fixed and determinate; the future is open and indeterminate. The arrows of time and causation point from past to future, not from future to past.

The Growing-Block view explains the difference between the fixed past and the open future in terms of ontological commitment: the view is committed to the (tenseless) existence of past objects and events, but not to the (tenseless) existence of future objects or events. It treats the present like the past, not like the future: present things are the last things the Growing-Block theory takes seriously.

The commitments of the Growing-Block theory change - or more precisely, increase - as time passes. Though the Growing-Block theory takes only the past and present seriously, it holds that in time, there will be more to the past and present, because more will have happened, even though the events

\footnotetext{
${ }^{0}$ Thanks to Jamin Asay, David Braddon-Mitchell, Mark Colyvan, Alison Fernandes, Patrick Greenough, Dominic Hyde, Jenann Ismael, Mark Jago, Rosanna Keefe, Kristie Miller, Jonathan Payne, Huw Price, Lionel Shapiro, Nicholas J.J. Smith, Agustin Rayo, and Richmond Thomason for their helpful questions and comments.
} 
that will happen are not ones to which the Growing-Block view is (tenselessly) committed.

Claims about the future create a puzzle for the Growing-Block theory. We ordinarily think that there are truths about the future - for instance, it is true that there will be a lunar eclipse on January 21, 2019. (Astronomers know that there will be a lunar eclipse on January 21, 2019, and what is known is surely true.) But if the future is unreal, then all claims about the future would seem to be trivially false.

It will not help to stipulate that all sentences about the future are trivially true or trivially truth valueless, instead of trivially false. The proposition that there will be a lunar eclipse on January 21, 2019 is true, and the proposition that the moon will be replaced with a hunk of green cheese on January 21, 2019 is false. Everyone - Growing-Block theorists included - should distinguish between truths about the future and falsehoods about the future.

We claim that although Growing-Block theorists must deny the reality of the future, they can still countenance non-trivial truths and falsehoods about future events. In the remainder of part 1, we develop a metaphysical and semantic framework for discussing modality in the context of the Growing-Block theory. This stage lays the groundwork for an account of future possibility, or feasibility, which can in turn be used to define a concept of future truth.

In part 2, we propose three ways in which Growing-Block theorists might deploy our modal framework to give semantics for sentences about the future. Each proposal is associated with a different non-classical logic. Rather than choose among these proposals, we state the advantages and disadvantages of each, and leave the reader to decide.

In part 3, we respond to two general problems, having to do with expressive power, that face three all of our semantic proposals. Finally, in part 4, we explain the advantages of the Growing-Block theory over similar theories proposed by Craig Bourne, Storrs McCall, and John MacFarlane.

Before we continue, one word about our starting point is in order. We will treat propositions as tensed - as having truth values relative to a time and to a possible world. We will use $\mathbf{P}$ and $\mathbf{F}$ as past and future operators on tensed sentences. These are to be read 'At some past time' and 'At some future time'

respectively. This clarification out of the way, we are ready to develop the Growing-Block theory in more detail.

\subsection{Time (Genuine and Ersatz)}

Our first task is to develop an ontology of possible worlds suited to the purposes of the Growing-Block theory. This ontology is a type of linguistic ersatzism: it presupposes that possible worlds are not concrete hunks of matter like the actual world, but descriptions of the world in a logically idealized language.

We follow the approach of Craig Bourne [2006, 52-54], who builds ersatz worlds from ersatz times. Just as an ersatz world is a complete representation of a world from a tenseless perspective, an ersatz time is a complete representation of a single time in a world. Each ersatz time is a maximal consistent set of 
tensed propositions which do not themselves contain any tense operators. (Ultimately, we will develop a way of assigning truth values to sentences containing tense operators - so the propositions contained in an ersatz time comprise only a proper subset of the propositions which are true at that time.) We take propositions to be interpreted sentences in an idealized logical language, and we will switch freely between talk of propositions and talk of sentences.

We will make some further assumptions about the structure of ersatz times. We assume that each ersatz time $e$ is associated with a vocabulary of names $D_{e}$, which (in a slight abuse of the use-mention distinction) we will call a 'domain'. The domains of different ersatz times may overlap partially, completely, or not at all. We assume that if $e$ contains a sentence of the form $\exists x \phi(x)$, then it also contains a sentence of the form $\phi(a)$ for some $a \in D_{e}$. (Both these assumptions are claims about what it is for $e$ to be a complete representation of a time: $e$ must specify exactly which things exist; and if it says that something satisfies a particular open sentence, then it also specifies which thing satisfies that open sentence.) Finally, we assume a "one name, one object" rule: each name has at most one referent, and refers to the same object across times and possible worlds, while each object has at most one name.

Since they are abstract representations, ersatz times contain no tables and chairs, donkeys and cats, or quarks and electrons. Nonetheless, they can be instantiated by concrete times: maximal simultaneous hunks of spatially connected stuff that do include tables, chairs, donkeys, cats, quarks, and electrons as parts. For a concrete time $t$ to instantiate an ersatz time $e$, we claim, is simply for $e$ to be a complete and true description of $t$. The concrete present instantiates one ersatz time; various concrete past times have instantiated other ersatz times. Still other ersatz times are not instantiated, and never have been.

The Growing-Block theorist should claim that concrete times (past and present) are ordered from earlier to later. We will assume that this ordering relation is total, transitive, irreflexive, and asymmetric, and that it has all four properties necessarily. Following Bourne [2006, 53-55], we can represent possible timelines as sets of ersatz times ordered by a total, transitive, irreflexive, and asymmetric relation $R .^{12}$ (The same ersatz time may appear more than once in the ordering.)

Having defined timelines, we can now explain what it is for a timeline to be instantiated. The whole of reality instantiates a timeline $T$ just in case there is a function $f$ mapping the ersatz times in $T$ onto concrete times such that

\footnotetext{
${ }^{1}$ Perceptive readers will note that we have just ruled out a number of apparent possibilities, including closed time-like curves and branching concrete universes. We are not at all convinced these apparent possibilities are genuine possibilities. We believe that our theory could be expanded to accommodate these scenarios, but to do so here would require adding to an already long and complex paper.

${ }^{2}$ In addition to the ordering relation $R$, one might think that there are meaningful distance relations between ersatz times. Given two times such that one is later than the other, it makes sense to ask how much later - an hour, a minute, a year? We will assume that facts about the distances between times supervene on the facts about which concrete times there are and how those times are ordered, and do not need to be added to possible timelines as extra information.
} 
(i) for every concrete time $t$, there is some $e \in T$ such that $f(e)=t$,

(ii) for every $e \in T, f(e)$ concretely instantiates $e$, and

(iii) for every $e, e^{\prime} \in T, e R e^{\prime}$ iff $f(e)$ is earlier than $f\left(e^{\prime}\right)$.

Timelines, then, are sequences of ersatz times. They are possible world histories, or put another way, they are ersatz possible worlds. The Growing-Block theorist may want to place additional restrictions on which timelines represent genuine metaphysical possibilities. For instance, perhaps the properties of objects must change in a more-or-less continuous fashion, so that no possible timeline can represent a person as having one head at one time and three heads at a time immediately afterward. (Note that this continuity constraint is available, but not mandatory.) Only a subset of the possible timelines will be physically possible, biologically possible, and so forth. Saying exactly which timelines are possible in which ways is a serious philosophical problem, but it is not a problem peculiar to the Growing-Block view. We will not address it here, except to make explicit our assumption that what is possible according to $T$ supervenes on the ersatz times $T$ contains, together with the ordering of those times.

\subsection{Truth (Relative and Absolute)}

Now that we have developed an ontology of possible worlds, our next step is to define truth at a possible world. Or rather, since we are working with tensed propositions, to define truth at a time at a possible world.

A complete account of truth will require a thorough discussion of how to treat the nonexistent future, and we will not be in a position to give such an account until part 2. In the meantime, though, we can give the beginnings of a definition of truth at a time in a timeline. These beginnings will help us to clarify the question of how to treat the nonexistent future, which in turn will help us to further clarify the concept of truth.

We will write $v_{e T}(\phi)$ for the truth value of $\phi$ according to $e$ in $T$. Defining truth for atomic propositions is straightforward.

- Where $p$ is an atomic proposition,

$$
v_{e T}(p)=\begin{array}{ll}
1 & \text { if } p \in e \\
0 & \text { if } p \notin e
\end{array}
$$

The definitions of truth-functional connectives are similarly straightforward.

- $v_{e T}(\neg \phi)=\begin{array}{ll}1 & \text { if } v_{e T}(\phi)=0 \\ 0 & \text { if } v_{e T}(\phi)=1\end{array}$

- $v_{e T}(\phi \wedge \psi)=\begin{array}{ll}1 & \text { if } v_{e T}(\phi)=1 \text { and } v_{e T}(\psi)=1 \\ 0 & \text { if } v_{e T}(\phi)=0 \text { or } v_{e T}(\psi)=0\end{array}$

- $v_{e T}(\phi \vee \psi)=\begin{array}{ll}1 & \text { if } v_{e T}(\phi)=1 \text { and } v_{e T}(\psi)=1 \\ 0 & \text { if } v_{e T}(\phi)=0 \text { or } v_{e T}(\psi)=0\end{array}$ 
We assume that the material conditional $\phi \supset \psi$ is equivalent to the disjunction $\neg \phi \vee \psi$, at least until section 2.3, where we present an alternative definition of the material conditional. We will assume that $\phi \equiv \psi$ is equivalent to the conjunction $(\phi \supset \psi) \wedge(\psi \supset \phi)$.

We can define tensed quantifiers $\exists$ and $\forall$, which range (at $e$ in $T$ ) over all and only the objects whose names belong to $D_{e}$.

- $v_{e T}(\exists x \phi x)=1 \quad$ if there is some $a \in D_{e^{\prime}}$ such that $v_{e T}(\phi x / a)=1$

- $v_{e T}(\forall x \phi x)=\begin{array}{ll}1 & \text { if for all } a \in D_{e}, v_{e T}(\phi x / a)=1 \\ 0 & \text { if for some } a \in D_{e}, v_{e T}(\phi x / a)=0\end{array}$

- $v_{e T}(\forall x \phi x)=\begin{aligned} & 0 \\ & 0\end{aligned}$ if for some $a \in D_{e}, v_{e T}(\phi x / a)=0$

We can also partially define tenseless quantifiers $\Sigma$ and $\Pi$, which range over everything there was, is, or will be.

- $v_{e T} \Sigma x(\phi x)=1$ if for some $e^{\prime}$ in $T$, there is an $a \in D_{e^{\prime}}$ such that $v_{e^{\prime} T}(\phi x / a)=1$

- $v_{e T} \Pi x(\phi x)=0 \quad$ if for some $e^{\prime}$ in $T$, there is an $a \in D_{e^{\prime}}$ such that $v_{e^{\prime} T}(\phi x / a)=0$

We have not fully defined $\Sigma$ or $\Pi$, because we have not yet established a way of assigning truth values to statements about the future. Where there is no object $x$ such that $\phi x$, it may be the case that there will be an object $x$ such that $\phi x$ (once more times have become instantiated by the Growing-Block), or it may not. In order to settle the matter, we will need to say more about the future.

We can also fully define the tense operator $\mathbf{P}$, and partially define the tense operator $\mathbf{F}$.

- $v_{e T}(\mathbf{P} \phi)=\begin{array}{ll}1 & \text { if } T \text { contains an } e^{\prime} \text { such that } e^{\prime} R e \text { and } v_{e^{\prime} T}(\phi)=1 \\ 0 & \text { else }\end{array}$

- $v_{e T}(\mathbf{F} \phi)=1$ if $T$ contains an $e^{\prime}$ such that $e R e^{\prime}$ and $v_{e^{\prime} T}(\phi)=1$

$\mathbf{F}$ is only partially defined for the same reason that $\Sigma$ and $\Pi$ are only partially defined. We've said nothing yet about how to ascertain $v_{e T}(\mathbf{F} \phi)$ when $T$ fails to contain an $e^{\prime}$ such that $e R e^{\prime}$ and $v_{e^{\prime} T}(\phi)=1$. We do not want to say that $v_{e T}(\mathbf{F} \phi)$ is invariably 0 under these circumstances, because we want to allow for the possibility that $\phi$ will become true in the future that does not yet exist.

We can introduce an additional class of tense operators - one for each temporal distance $d$. (We will write the distance from $e$ to $e^{\prime}$ as positive where $e R e^{\prime}$, and as negative where $e^{\prime} R e$.)

$$
\text { - } v_{e T}\left(\mathbf{P}_{d} \phi\right)=\begin{aligned}
& 1 \quad \begin{array}{l}
\text { if } T \text { contains an } e^{\prime} \text { such that } e^{\prime} R e, \\
\text { the distance from } e \text { to } e^{\prime} \text { is } d, \\
\text { and } v_{e^{\prime} T}(\phi)=1 \\
\text { else }
\end{array}
\end{aligned}
$$


1 if $T$ contains an $e^{\prime}$ such that $e R e^{\prime}$, the distance from $e$ to $e^{\prime}$ is $d$,

- $v_{e T}\left(\mathbf{F}_{d} \phi\right)=\quad$ and $v_{e^{\prime} T}(\phi)=1$

0 if $T$ contains an $e^{\prime}$ such that $e R e^{\prime}$, the distance from $e$ to $e^{\prime}$ is $d$, and $v_{e^{\prime} T}(\phi)=0$

$\mathbf{F}_{d}$ is only partially defined, for the same reason that $\mathbf{F}$ was only partially defined.

Since $\Sigma, \Pi, \mathbf{F}$, and the $\mathbf{F}_{d}$ operators are only partially defined, the valuation function as a whole is only partially defined. The fragment of the language containing only atomic sentences, $\wedge, \vee, \neg$, the quantifiers, and $\mathbf{P}$ behaves classically. But the valuation function as a whole is non-classical. We will discuss three different (non-classical) ways of extending the valuation function in sections 2.1-2.3.

In addition to the concept of truth at a time in a timeline (which we have yet to fully define), we can define a number of less relativized types of truth: truth at a time, truth in a timeline, and truth, full stop. There is one timeline that represents the world as it really is - that is, it correctly represents all the times that concretely exist, and it does not represent the world as containing any times that don't concretely exist. This is the actualized timeline. We can say that what is true at a (concrete) time $t$ is simply what is true according to the ersatz time $e$ in the actual timeline (an ordered set of ersatz times) instantiated by a concrete time $t$ in the actual world (a temporally ordered hunk of concrete future stuff).

To define truth at a timeline, we will need the concept of an absolute present. Many theorists of time treat 'present' as an indexical, so that a token of 'it is raining at present' is true just in case it is raining at the time when the sentence is uttered. But the Growing-Block theorist can also pick out a non-indexical or absolute present: the last time in existence. Furthermore, each timeline has its own (ersatz) absolute present: the last time in it. The ersatz absolute present in a timeline $T$ would, if $T$ were actualized, be instantiated by the concrete absolute present.

What is true at a timeline $T$ is simply what is true at $T$ 's absolute present. Some complete timelines will have no absolute present, since they will have no last time. There is nothing that is true according to these timelines (though there are still propositions that are true at times in them).

What is true full stop is what is true at the absolute present in the actualized timeline. That is to say, what is true full stop is what is true according to the concrete hunks of stuff that exist at the current stage of the Growing-Block. We have four concepts of truth in all, corresponding to cells in the following table.

\begin{tabular}{cc|c|c|} 
& \multicolumn{2}{|c}{ Timeline } \\
& Relative & Absolute \\
\hline \multirow{2}{*}{ Time } & Relative & Truth at $e$ in $T$ & Truth at $t$ \\
\cline { 2 - 3 } & Absolute & Truth in $T$ & Truth \\
\hline
\end{tabular}


The cells on the left-hand side of the table correspond to types of truth that are wholly relative to representations, while the cells on right-hand side correspond to types of truth that are somehow grounded in concrete existence. The cells in the top row correspond to tensed types of truth, while the cells in the bottom row correspond to tenseless types of truth. (Notice that we have used a single, tensed type of proposition throughout, but that our tensed propositions admit of both tensed and tenseless truth.)

Given our fourfold definition of truth, how shall we define validity? A valid sentence is necessarily true, while a valid argument is one that is necessarily truth-preserving. We need a univocal way of understanding 'true' and 'truth' (and also a univocal way of understanding 'necessarily').

Which sentences and arguments are valid should not depend on what concretely exists. Rather, questions of validity must be evaluated by considering all possibilities - both those that are concretely instantiated and those that are not. Possibilities, in our framework, are timelines. Therefore, we should define a valid argument as as one that preserves one of the timeline-relative types of truth on the left-hand side of the matrix: either truth at $e$ in $T$ or truth in $T$. We have two appealing conceptions of validity:

Validity $_{e T} \Phi \models_{e T} \Psi$ just in case, for every time $e$ and timeline $T$, if $v_{e T}(\phi)=1$ for all $\phi \in \Phi$, then $v_{e T}(\psi)=1$ for at least one $\psi \in \Psi$.

Validity $_{T} \Phi \models_{T} \Psi$ just in case, for every time $e$ and timeline $T$ such that $e$ is the absolute present in $T$, if $v_{e T}(\phi)=1$ for all $\phi \in \Phi$, then $v_{e T}(\psi)=1$ for at least one $\psi \in \Psi$.

Validity $_{e T}$ is the stronger notion: an argument or sentence that is valid ${ }_{e T}$ must also be $\operatorname{valid}_{T}$, but the converse does not hold. ${ }^{3}$

We will take validity to be validity ${ }_{e T}$, rather than the weaker validity ${ }_{T}{ }^{4}$ Likewise, we will take necessity to be truth at every time in every timeline - not just truth in every timeline.

The distinctions among the four relativized types of truth can be brought to bear on a recent objection to the Growing-Block view. We discuss this objection and our solution to it in the next section.

\subsection{How Do We Know it is Now Now?}

Craig Bourne [2006] and David Braddon-Mitchell [2004] have independently raised the concern that, if there are two interpretations of 'the present' on the Growing-Block view (viz. the indexical and the absolute), then it is possible

\footnotetext{
${ }^{3}$ The following example shows that validity ${ }_{T}$ does not entail validity $_{e T}$. We might define an "absolute present" operator @ such that

- $v_{e T}(@ \phi)=\begin{array}{ll}1 & \text { if } e^{\prime} \text { is the absolute present in } T \text { and } v_{e^{\prime} T}(\phi)=1 \\ 0 & \text { else }\end{array}$

The sentence $\phi \supset @ \phi$, and the inference from $\phi$ to @ $\phi$, are $\operatorname{valid}_{T}$ but invalid $\operatorname{li}_{e T}$.

${ }^{4}$ Still other interpretations of validity are possible in the supervaluationist framework we develop in section 2.1; see [Varzi, 2007] for a detailed discussion.
} 
that, say, Marie Curie might ask if, in addition to being at the indexical present (i.e. being at the time she is at) she is at the absolute present (i.e. at the time succeeded by nothing). If we accept that Marie Curie could ask such a question, despite not being at the absolute present, then we must explain why Marie Curie would be wrong to think she is at the absolute present, while we are right to think we are at the absolute present.

One solution, put forward by Peter Forrest, is to deny the possibility of Marie Curie asking such a question, so long as she is located in the past. According to him "the past, although real, is lifeless and (a fortiori?) lacking in sentience [Forrest, 2004, 358]." More generally, "Life and sentience are... activities not states. Activities only occur on the boundary of reality, while states can be in the past [Forrest, 2004, 359]." For Forrest, the distinction between the living present and the dead past is grounded in their different causal propertiesactivity requires a 'causal frisson' that can occur only at the absolute present.

We think that the distinction between the living present and the dead past is better understood in terms of the distinction between what is true at a past time (i.e. what is true according to the ersatz time $e$ that corresponds to $t$ in the actualized timeline), and what is simply true (i.e. true at the absolute present in the actualized timeline). This distinction is meant to be applied concerning not the content of Marie Curie's beliefs, but our attribution of those beliefs to her. It is true at some past time (e.g. 1903) that Marie Curie falsely believes she is (absolutely) present, but it is not true (full stop) that she falsely believes

she is (absolutely) present. This is because it is not true (full stop) that Marie Curie believes anything at all. In the actual world, at the absolute present, Curie is dead. Dead people lack beliefs.

Whether the concept of absolute truth aligns with any causal concept is a further question, and one that the authors of this essay disagree about. One of us thinks Forrest's concept of a causal frisson captures something important about the sense in which the present is ontologically privileged; the other thinks that it is unlikely to satisfy any of the useful roles carved out for causation in metaphysics and the philosophy of science.

\section{$1.4 \quad$ Feasibility}

So far, timelines have behaved somewhat like the possible worlds of Robert Merrihew Adams [1974]. We should, however, mark several important disanalogies between timelines and traditional possible worlds. First, unlike traditional possible worlds, timelines are not sets of propositions, but ordered sets of such sets. Second, discerning what is true at a time (i.e., a set of tensed propositions) in a timeline, unlike discerning what is true at a traditional possible world (i.e., a set of tenseless propositions), is not simply a matter of checking to see which propositions the relevant set contains. Every proposition contained in a time in a timeline is true at that time in that timeline, but a proposition may be true (within a timeline) at times that do not contain it.

Third, unlike traditional possible worlds, timelines stand in interesting parthood relations. In formal terms, we can say that timeline $T$ is an initial segment 
of timeline $T^{\prime}$, or that $T^{\prime}$ is an extension of $T$, just in case

(a) every ersatz time in $T$ also occurs in $T^{\prime}$,

(b) if $e R e^{\prime}$ in $T$, then $e R e^{\prime}$ in $T^{\prime}$, and

(c) if $e R e^{\prime}$ in $T^{\prime}$, then either $e R e^{\prime}$ in $T$, or for all $e^{*} \in T, e^{*} R e^{\prime}$ in $T^{\prime}$.

(We could define final-segment and middle-segment relations between timelines in a similar way, but the initial-segment relation is the one that will turn out to be most theoretically useful.)

With the apparatus set forth so far, the Growing-Block theorist is equipped to start talking about fixed past and the open future. The actual world concretely instantiates a timeline - the actualized timeline. There are a number of physically possible extensions of the actualized timeline. (Call these feasible timelines.) Feasible timelines, like the actualized timeline, correctly represent the denizens the actual world. Furthermore, like the actualized timeline, they evolve in a way compatible with the dynamic physical laws that govern the universe. So in a sense, they accurately represent the world - that is, everything in the world, they represent accurately. But unlike the actualized timeline, they represent some things that never happened. So in another sense, they fail to accurately represent the world - they represent it as containing things it does not in fact contain.

Feasible timelines are possible timelines, in some very strong sense of "possible". For all that the world determines, any feasible timeline might be actualized in the future, but the world does not determine which feasible timeline will be actualized in the future.

Feasibility can be understood as a possibility relation among timelines. "It is feasible that" behaves as a type of possibility operator, and its dual as a necessity operator. We will assume that the feasibility relation is transitive (what is feasibly feasible is feasible) and reflexive (every timeline is feasible relative to itself). Furthermore, feasibility is antisymmetric (no two distinct timelines are feasible relative to each other). The antisymmetry of the feasibility relation follows from the antisymmetry of the extension relation, together with the fact that what is feasible relative to a timeline must be an extension of it.

A set of feasible timelines for a time can be represented as a tree structure, like the one in figure 1. 


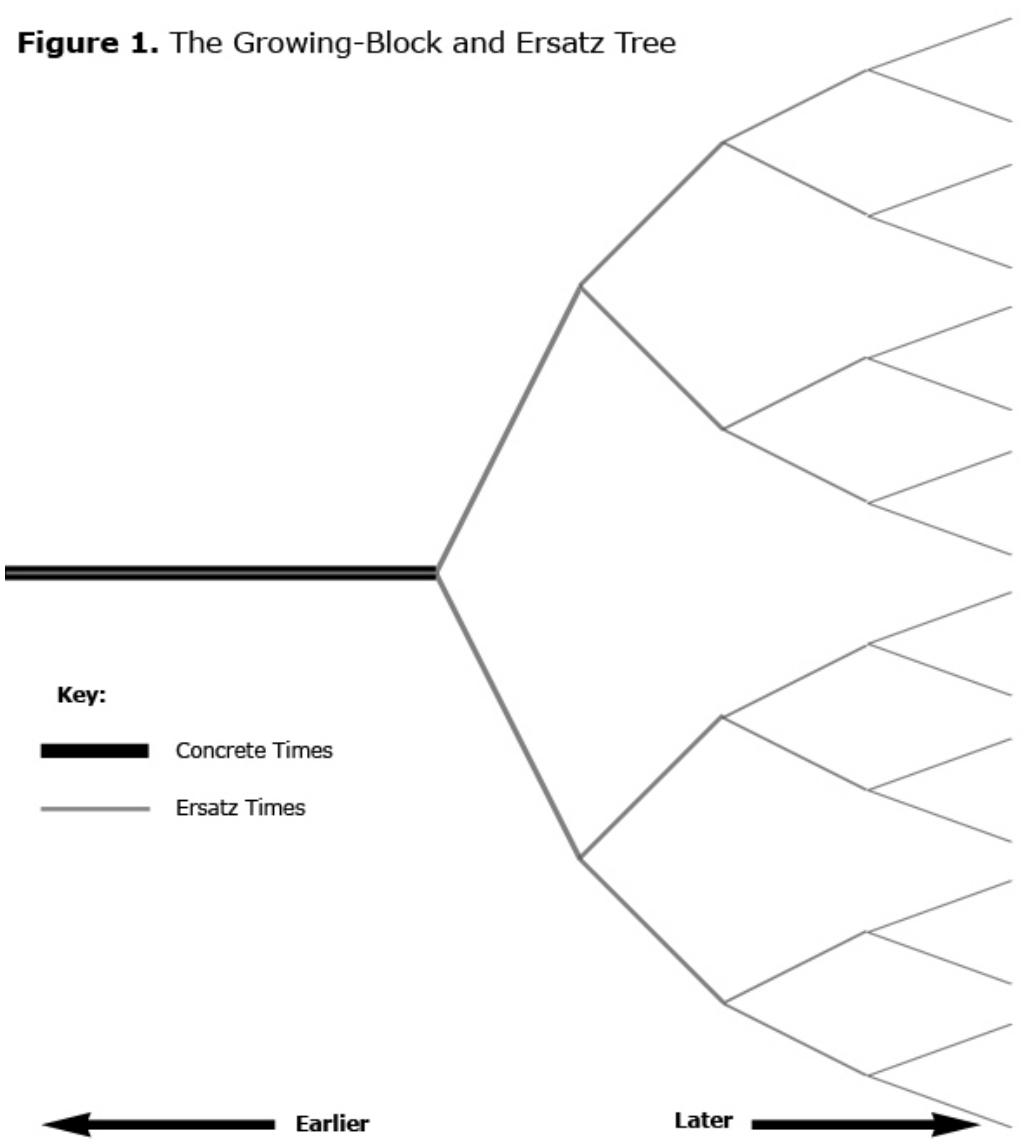

Notice that figure 1 depicts a great many timelines, since any initial segment of a timeline is itself a timeline. Some of those timelines, which represent particular kinds of possibilities, will play a special role in the semantics of sentences about the future. We can make the following distinctions between three types of timelines.

Some timelines end in a way that (together with the laws of nature) precludes any future happenings; they contain a final "big crunch", after which all space, time, and matter disappear, or their laws of nature permit certain boundless span of time - which is filled up with events that carry on indefinitely - and nothing more. Call these timelines complete. Other timelines seem to end abruptly, with tigers frozen mid-leap, the planets stopped in their orbits, and human actors posed in lifelike tableau. The laws of nature require that these timelines go on into the future; call them incomplete. Finally, there may be timelines that the laws of nature permit, but do not require, to extend into the future. Call these semi-complete.

We will leave open the question of how to distinguish among the three types of timelines, the question of whether there are any complete timelines, and the question of whether there are any semi-complete timelines. We will, however, 
assume that every timeline has either a feasible complete extension or a feasible semi-complete extension - that it is possible to tack on enough time to reach an acceptable stopping point.

The concepts of completeness and semi-completeness are crucial because they are linked to the concept of an exhaustive description-one that details everything that ever has happened, is happening, or will happen. A complete or semi-complete timeline can (in some appropriate nomological sense) be exhaustive in this sense, while an incomplete timeline cannot. When it comes to assigning truth values to sentences about the future, based on a timeline, it will be important to know whether the timeline is exhaustive - whether it catalogues everything that will be, in addition to everything that is.

The terms 'complete' and 'incomplete' have logical connotations: a classically complete valuation function is one that assigns value 0 or 1 to every sentence in some language, while a classically incomplete valuation function fails to assign values to some some sentences in the language. We welcome these connotations: it will turn out that every complete timeline is associated with a classically complete valuation function, incomplete timelines tend to be associated with classically incomplete valuation functions, and semi-complete timelines play two roles - one in which they must be associated with classically complete valuation functions, and one in which they may be associated with classically incomplete valuation functions.

\section{Evaluating Future Contingents}

Consider the tensed propositions expressed by the following sentences.

(1) Exactly one day into the future, there will be a sea battle. $\left(\mathbf{F}_{d} S\right)$

(2) Exactly one day ago, there was a sea battle. $\left(\mathbf{P}_{d} S\right)$

(3) Exactly one day into the future, it will be the case that: either there is a sea battle, or there is not. $\left(\mathbf{F}_{d}(S \vee \neg S)\right)$

(4) Either there will be a sea battle exactly one day into the future, or there will be no sea battle exactly one day into the future. $\left(\mathbf{F}_{d} S \vee \mathbf{F}_{d}(\neg S)\right)$

(5) Either there will be a sea battle exactly one day into the future, or it is not the case that there will be a sea battle exactly one day into the future. $\left(\mathbf{F}_{d} S \vee \neg \mathbf{F}_{d}(S)\right)$

(6) Someone will win tomorrow's lottery drawing (though not anyone in particular $).\left(\mathbf{F}_{d}(\Sigma x L x)\right)$

(7) There is someone (in particular) who will win tomorrow's lottery drawing. $\left(\Sigma x\left(\mathbf{F}_{d} L x\right)\right)$

How should one ascertain the truth values of these propositions? Do all of them have truth values? Do any? 
One option for the Growing-Block theorist is to claim that, since all of the sentences except (2) make apparent reference to a future that does not, strictly speaking, exist, all except (2) are either necessarily false or necessarily truth valueless. We think this option should be rejected. (1) looks like be the sort of proposition that can be known, at least under the right circumstances. If I know that the ships have already been launched on a collision course, and that there is no nomologically possible way of preventing the battle available in my circumstances, then I appear to be in a position to know (1). But surely what can be known can be true.

This argument is not unanswerable. Someone who believes that all the example sentences but (2) are truth valueless might reply that when one knows the ships have been launched beyond recall, one's attitude toward (1) is properly described not as knowledge, but as justified belief. (There will have to be some further story about what justified belief amounts to when the proposition believed is truth valueless, and knowably truth valueless.) But on the face of it, there appear to be circumstances in which (1) true, which can usefully be contrasted with circumstances in which (1) is false (e.g., circumstances in which no one has any ships, and it is not physically possible to make any by tomorrow).

So (1) seems to be true at some times in some timelines, and false at other times in other timelines. Are there also times when (1) is neither true nor false? The Growing-Block theorist has good reasons to say "yes". Sometimes, the past, the present, and the laws of nature are not enough to settle whether there will be a sea battle one day into the future. ${ }^{5}$ But according to the Growing-Block theory, there is nothing but the past, the present, and the laws of nature. If something is not settled by the past, the present, and the laws of nature, then it is not settled. Where nothing in the world settles whether a sentence is true or false, that sentence must not have a truth value - there is no truth without some sort of truthmaking.

Again, our argument is not unanswerable. Roy Sorensen [2001] and Patrick Greenough [2008] defend the position that all propositions are either true or false, that some true propositions are not made true by anything in the world, and that cases of indeterminacy are cases of truthmaker gaps rather than truth value gaps. The Sorensen-Greenough view has a notable payoff: its proponents can keep classical logic, while proponents of truth value gaps must give classical logic up. Still, contingent truths that are not grounded in reality seem puzzling and spooky. We think they are so spooky that on balance, it is best to assume that (1) is sometimes truth valueless (as well as sometimes true and sometimes false). Hardcore adherents of the Sorensen-Greenough approach, however, can re-interpret our discussion of truth and falsity as a discussion of grounded truth and grounded falsity.

(2), unlike (1), is about past events. The past-no matter what it was likeseems to be enough to fix the truth value of (2). ${ }^{6}$ This suggests that unlike (1),

\footnotetext{
${ }^{5}$ Even if the actual world is deterministic, we would not want to rule out the possibility of indeterministic worlds.

${ }^{6}$ Here and throughout, we ignore the complications caused by unrelated phenomena such as vagueness and the liar. A theory that addressed these complications might replace our
} 
(2) is always either true or false according to the Growing-Block theory. The possible indeterminacy of (1), when taken together with the necessary determinacy of (2), is one way of cashing out the idea that the future is open while the past is fixed.

Sentences (3)-(5) all appear to be logical consequences of (1). We will assume that appearances are right, although they could conceivably be disputed. (Disputation is easiest in the case of (3), since (4) and (5) follow from (1) by tautological consequence alone.) One might wonder whether in addition to being entailed by (1), (3)-(5) are logically valid - that is, whether they are entailed by the empty set. (3) looks valid at first glance - it is simply an instance of the law of excluded middle embedded in the scope of a future operator. And isn't tomorrow bound to obey the law of excluded middle, just like yesterday and today? On second thought, though, what if there is no tomorrow? (3) is arguably false at the last moment of the universe.

(4) seems to say something stronger than (3) - not just that the future obeys the law of excluded middle, but that it is determined by the past and the present, at least as far as sea battles are concerned. For (4) is a disjunction, and each of its disjuncts entails that the future is determined, at least as far as sea battles are concerned. One could object to the inference from the claim that each of (4)'s disjuncts entails that the future is determined to the claim that (4) itself entails that the future is determined. The supervaluationist view we discuss in section 2.1 takes this objection seriously. Still, there is at least a prima facie case for thinking that (4) might not be true, even when (3) is.

(5) is a classical tautology. This suggests that (5) is valid. But to all three of the proposals we consider, there are situations where the sentences $\mathbf{F}_{d} S$ and $\neg \mathbf{F}_{d} S$ are both untrue (that is, not true - either false or indeterminate). If (5) is valid, then it is sometimes true without having a true disjunct - a puzzling state of affairs!

(6) says simply that a prize will be given out tomorrow. (7) appears to say something stronger - that the lottery is a fix. The relationship between (6) and (7) is closely analogous to the relationship between (3) and (4). (3) says that a particular disjunction will be true tomorrow, while (4) says that one of its disjuncts will be true tomorrow. Likewise, (6) says that a particular existentially quantified statement will be true (of something that did, does, or will exist) tomorrow, while (7) says that there did, does, or will exist something that will witness that existentially quantified statement tomorrow. (4) and (7) seem to claim that certain questions (whether there will be a sea battle, who will win the lottery) are settled, while (3) and (6) seem not to make such claims.

We have chosen to translate both (6) and (7) using the tenseless quantifier $\Sigma$ rather than the tensed quantifier $\exists$. Our translation of (6) does not require that the winner of tomorrow's lottery be someone who will exist tomorrow. (For all the logic says, the winner may exist today but be dead by tomorrow's drawing, or may not be born until several years into the future.) Likewise, our translation

ersatz times - assumed to be classical-with ersatz times that were complete and consistent by the lights of some suitable non-classical logic. 
of (7) does not require that the future winner of tomorrow's lottery be someone who exists today. (For all the logic says, the winner may be dead already, or not yet born.) The aim of this choice is simply to avoid the complications that would result from changes in the domain of quantification.

We will consider three proposals for assigning truth values to sentences like (1)-(7): a supervaluationist proposal, a proposal inspired by Lukasiewicz's threevalued logic, and an intuitionist proposal. Each proposal has different strengths and requires different tradeoffs. We endorse the intuitionist proposal, but think the other two proposals are serious contenders worthy of attention.

Proponents of any non-classical logic must reject some intuitively plausible claims. All of the following claims are highly plausible, but there are numerous incompatibilities among them, which illustrate the tradeoffs available to the Growing-Block theorist.

(a) There are some propositions such that (it is at least possible that) neither they nor their negations are true.

(b) A disjunction can be true only if one of its disjuncts is.

(c) All classical tautological inferences are valid.

(d) All other things being equal, truth values should be assigned to as many propositions as possible.

(e) A language for describing the open future should be sufficiently powerful to include an expression for determinate truth.

(f) Reductio ad Absurdum, Contraposition, and Conditional Proof are valid natural deduction inferences.

(g) Truth is determinate truth: a sentence is true just in case it is guaranteed by history together with the laws of nature.

(h) Truth is disquotationial: if a truth operator $\operatorname{Tr}$ is introduced into the language, every instance of the schema $\phi \equiv \operatorname{Tr} \phi$ should be valid. ${ }^{7}$

All three of our proposals are committed to (a). They count (1) as sometimes true, sometimes false, and sometimes indeterminate. They also agree in counting (2) as always either true or false, and (3) as true in circumstances where the universe is guaranteed to persist until tomorrow, false in circumstances where the universe is guaranteed not to persist until tomorrow, and indeterminate otherwise.

All three of our proposals entail the truth of (a). For reasons we discussed at the beginning of this section, Growing-Block theorists should accept (a). There are (or could be) propositions about the future whose truth values are not settled by the past, the present, and the laws of nature. For Growing-Block theorists,

\footnotetext{
${ }^{7}$ Some exceptions will have to be made in cases of self-reference; we ignore these complications here.
} 
this means that there are (or could be) propositions whose truth values are not settled at all.

Philosophers who accept (a) must make tradeoffs elsewhere. First, they must choose between giving up (b) and giving up (c). By (a), there is some disjunction $\phi \vee \neg \phi$, neither of whose disjuncts is true - (5) is such a disjunction under some possible circumstances. By (c), (5) is sometimes untrue. But since (5) is tautologically valid, by (b) it is always true. So rejecting either (b) or (c) is mandatory.

The supervaluationist proposal gives up (b) and keeps (c). ${ }^{8}$ It treats (3) and (4) as equivalent, and (6) and (7) as equivalent. The Eukasiewicz and intuitionist proposals, on the other hand, give up (c) and keep (b). They are able to treat (4) as stronger than (3) and (6) as stronger than (7).

(d) need not be accepted or denied wholesale, but can be accepted to a greater or a lesser extent, depending on how important one thinks it is to assign truth values to as many sentences as possible. If we treat (d) as a desideratum for theories, the supervaluationist semantics does better than the intuitionist semantics, which in turn does better than the Łukasiewicz semantics. All three proposals are outdone by classical logic, but we think the cost of classical logic for the Growing-Block theorist - spooky truths without truthmakers - is excessive.

In addition to the tradeoff between (b) and (c), and the decision about how seriously to take (d), accepting (a) necessitates a tradeoff between (e) and (f). In any sufficiently powerful semantics that allows for truth value gaps, one can formulate propositions for which Reductio ad Absurdum, Contraposition, and Conditional Proof fail. (A reminder: these three inferences are as follows:

Reductio ad Absurdum If $\phi \vdash \perp$, then $\vdash \neg \phi$.

Contraposition If $\phi \vdash \psi$, then $\neg \psi \vdash \neg \phi$.

Conditional Proof If $\phi \vdash \psi$, then $\vdash \phi \supset \psi$.

Since entailment is transitive and $\neg \perp$ follows from the empty set, Reductio ad Absurdum is the special case of Contraposition where $\psi=\perp$.)

The above inferences fail in three-valued logics because they rely on the assumption that there is no gap between truth and falsity. If $\phi$ entails $\psi$, then there is no time in any timeline where $\phi$ is true and $\psi$ is false. But where there are truth value gaps, $\phi$ 's entailing $\psi$ is compatible with there being times in timelines at which $\phi$ is indeterminate and $\psi$ is false. In any sufficiently powerful language, one will be able to define a $\phi$ and a $\psi$ such that $\phi$ entails $\psi$, and $\phi$ may be indeterminate where $\psi$ is false. Possibilities in which $\phi$ is true and $\psi$ is not true can be redescribed as possibilities in which $\neg \psi$ is true and $\phi$ is not true. In such possible circumstances, $\phi \supset \psi$-a conditional with a true antecedent and an indeterminate consequent - should not count as true. Even if $\phi$ entails $\psi, \neg \phi$ does not necessarily entail $\neg \psi$, nor is the conditional $\phi \supset \psi$ necessarily valid.

\footnotetext{
${ }^{8}$ At least, it keeps (b) in the sense that it validates all classically valid single-conclusion arguments. Classically valid multiple-conclusion arguments are supervaluationistically invalid.
} 
Finally, accepting (a) means trading off between (g) and (h). If (a) is correct, then there are some sentences that are indeterminate - not false, but not true. It is false that these sentences are determinate. So, where $\phi$ is a proposition with indeterminate truth value, and $\triangle$ is a determinacy operator, $\Delta \phi$ is false. When a biconditional has an indeterminate sentence on one side and a false sentence on the other, the entire biconditional should not count as true - it is either indeterminate or false. (This claim could be disputed, but it holds according to all the interpretations of the biconditional we consider.) Therefore, where $\phi$ is indeterminate, $\Delta \phi$ is false, and so the biconditional $\phi \equiv \Delta \phi$ is not true.

\subsection{The Supervaluationist Semantics}

Richmond Thomason [1970] has proposed a supervaluationist semantics for future contingents, which can easily be adapted to the suit the Growing-Block theorist's ontology. Our discussion of Thomason will be somewhat technical, but the gist of his theory is this: $\phi$ is true if every possible future course of events will make $\phi$ true, false if every possible future course of events will make $\phi$ false, and indeterminate otherwise.

More technically, for Thomason sentences receive truth values at times in model structures. Each of Thomason's times is associated with a classical valuation function over sentences without tense operators - our ersatz times are therefore well suited to play the role of Thomason's times. A model structure, for Thomason, is a set of times partially ordered by a relation $R$. Thomason assumes that $R$ is "treelike" - meaning that each time has a unique past, or more formally, that for all times $e, e^{\prime}$, and $e^{*}$ in the model structure, if $e^{\prime} R e$, $e^{*} R e$, and $e^{\prime} \neq e^{*}$, then either $e^{\prime} R e^{*}$ or $e^{*} R e^{\prime}$.

Truth at a time in a model structure is defined in terms of truth at a time in a history - a maximal set of times $h$ such that for any two distinct times in the set, one bears the $R$ relation to the other. (One can picture histories as branches of the tree stretching all the way from root to leaf-tip.) A proposition $\phi$, says Thomason, is true at $e$ according to a model structure $M$ if $v_{e h}(\phi)=1$ for every $h$ in $M$ such that $e \in h$, false at $e$ according to $M$ if $v_{e h}(\phi)=0$ for every $h$ in $M$ such that $e \in h$, and indeterminate at $e$ in $M$ otherwise.

The next step, then, is to define truth at a time in a history. Where $e$ is any time and $h$ is any history such that $e \in h, e$ and $h$ are associated with a classical valuation function $v_{e h}$, built up in a familiar fashion. For any atomic sentence $p$,

$$
\text { - } v_{e h}(p)=\begin{array}{ll}
1 & \text { if } p \text { is true according to } e \\
0 & \text { else }
\end{array}
$$

Thomason adds the usual clauses for the truth-functional connectives (he does not discuss quantification) and the following clauses for tense operators $\mathbf{P}$ and F.

$$
\text { - } v_{e h}(\mathbf{P} \phi)=\begin{array}{ll}
1 & \text { if } h \text { contains an } e^{\prime} \text { such that } e^{\prime} R e \text { and } v_{e^{\prime} h}(\phi)=1 \\
0 & \text { else }
\end{array}
$$


- $v_{e h}(\mathbf{F} \phi)=\begin{array}{ll}1 & \text { if } h \text { contains an } e^{\prime} \text { such that } e R e^{\prime} \text { and } v_{e^{\prime} h}(\phi)=1 \\ 0 & \text { else }\end{array}$

Notice that so far, the definition of $v_{e h}$ looks exactly like the definition of $v_{e T}$, except that the $\mathbf{F}$ operator is fully rather than partially defined. $\mathbf{F}(\phi)$ is false at $e$ in $h$ whenever it is not true at $e$ in $h$. Before we go on defining $v_{e h}$ for a larger range of operators and quantifiers, it is worth pausing to think about how Thomason's system maps onto ours.

We have already said that Thomason's times correspond to our ersatz times. In order to finish adapting Thomason's semantics to our ontology, we will need to find objects in our ontology to play the role of his model structures and histories.

Sets of the form $\left\{T^{\prime}: T^{\prime}\right.$ is a feasible extension of $\left.T\right\}$ are ideally suited to play the role of model structures. These sets, as we pointed out in section 1.4, have a tree-like structure. Furthermore, they are exactly the right sorts of things to relativize truth to. Each set is uniquely associated with a timeline, and timelines represent possibilities. Since our ultimate aim is a concept of something like truth in a possible world, we want to relativize truth to timelines (or to entities that admit of a natural one-to-one mapping to timelines).

Given a timeline and the tree structure consisting of its feasible extensions, we will need to pick out some entities to serve as its histories. Histories in Thomason's system must be something like exhaustive catalogues of events. This must be the underlying rationale for the semantic rule that treats $\mathbf{F}(\phi)$ as false at $e$ in $h$ whenever $h$ fails to contain an $e^{\prime}$ later than $e$ at which $\phi$ is true. If $h$ does not explicitly represent the universe as containing a time at which $\phi$, then $h$ represents the universe as not containing a time at which $\phi$.

Complete timelines are well-suited to play the role of histories in Thomason's system. They are exhaustive catalogues of events, or to put it another way, they describe ways the Growing Block might finish up, if it were allowed to grow until it could grow no more.

What about semi-complete timelines? There seem to be two possible ways of considering them. On the one hand, one can consider them under the assumption that they are exhaustive catalogues of the universe's events - complete descriptions of the way the Growing Block will end up. (After all, it is nomologically possible for the Growing Block to grow until it actualizes a semi-complete timeline, and then stop growing.) In other words, semi-complete timelines can be considered as histories. This is the appropriate stance if one is thinking from the perspective of a particular timeline, considering all possible exhasutive ways things might go next. The semi-complete extensions of the original timeline are indeed exhaustive ways things might go next.

On the other hand, one can take semi-complete timelines at face value, not assuming that they are exhaustive. (After all, it is nomologically possible that the Growing Block to grow until it actualizes a semi-complete timeline, and then keep growing.) This is the appropriate stance to take if one is thinking from the perspective of a semi-complete timeline, wondering what is true according to it. It is a way of thinking of the semi-complete timeline in itself. 
Depending on how we think of a semi-complete timeline - either as a history or in itself - different sentences will come out as true at various times in that timeline. Where $e$ is a time in a semi-complete timeline $T$, what is really true at $e$ in $T$ is what is true at $e$ according to $T$ considered in itself. But for the purposes of divining what is true at times in other timelines, it may be necessary to consider what is true at $e$ according to $T$ considered as a history.

One must treat complete timelines as histories, since is is nomologically necessary that they be exhaustive. Unlike the case of semi-complete timelines, there is no difference between considering complete timelines as and considering them in themselves. And one may not treat incomplete timelines as historiesthey cannot be exhaustive catalogues of the world's events. Growing Blocks that actualize incomplete timelines will have an irresitible impetus to keep growing adding more to the catalogue of the world's events. Incomplete timelines are not exhaustive, and should only be considered in themselves.

Since histories are timelines considered as exhaustive representations of the universe, we can add the following clauses to the definition of $v_{e h}$.

$$
\begin{aligned}
& 1 \text { if } h \text { contains an } e^{\prime} \text { such that } e R e^{\prime} \text {, } \\
& \text { - } v_{e h}\left(\mathbf{F}_{d} \phi\right)=\quad \text { the distance from } e \text { to } e^{\prime} \text { is } d \text {, } \\
& 0 \text { else } \\
& \text { - } v_{e h} \Sigma x(\phi x)=\begin{array}{ll}
1 & \text { if for some } e^{\prime} \text { in } h \text {, there is an } a \in D_{e^{\prime}} \text { such that } v_{e^{\prime} h}(\phi x / a)=1 \\
0 & \text { else }
\end{array} \\
& \text { - } v_{e h} \Pi x(\phi x)=\begin{array}{l}
0 \quad \begin{array}{l}
\text { if for some } e^{\prime} \text { in } h, \text { there is an } a \in D_{e^{\prime}} \\
\text { such that } v_{e^{\prime} h}(\phi x / a)=0 \\
0 \quad \text { else }
\end{array}
\end{array}
\end{aligned}
$$

So truth for sentences whose main operators are $\mathbf{F}, \mathbf{F}_{d}, \Sigma$, and $\Pi$ is defined for histories exactly as it was defined for incomplete and semi-complete timelines considered in themselves. But for histories, the definition of falsity is more extensive: sentences are false whenever they are not true. In incomplete timelines, and in semi-complete timelines considered in themselves, there may be truth value gaps; in histories there are none

How do propositions about the future get their truth values in incomplete timelines, or in semi-complete timelines considered in themselves? For us, each timeline is associated with a model structure - the set of its complete or semicomplete extensions. We can say that $\phi$ is true at $e$ in $T$ just in case it is true at $e$ in the model structure associated with $T$. Now it only remains to interpret the concept of truth in a model structure, according to our conception of model structures as sets of extensions of individual timelines.

Every model structure contains histories: complete timelines and semi-complete timelines which can be considered as histories. So, mapping our ontology onto Thomason's semantics yields the following inheritance clause: 
- Where $T$ is an incomplete timeline, or a semi-complete timeline considered in itself,

1 iff for every extension $h$ of $T$ such that $h$ is either a complete timeline or a semi-complete timeline treated as a history,

$v_{e T}(\phi)=\quad$ and $v_{e h}(\phi)=1$

and $v_{e h}(\phi)=1$
iff for every extension $h$ of $T$ such that $h$ is either

a complete timeline or a semi-complete timeline

treated as a history,

and $v_{e h}(\phi)=0$

If the supervaluatinist semantics is to be consistent, the new inheritance clause cannot assign values to compounds which are incompatible with the values assigned to those compounds by the definitions of conjunction, negation, and the tense operators. Fortunately, the types of sentences introduced so far have a convenient heredity property (proved in the appendix). Any sentence that receives value 0 or value 1 at $e$ in $T$ must receive the same value at $e$ in every feasible extension of $T$. So the inheritance clause cannot generate any inconsistency in the valuation function - at least for the types of sentences considered so far. (For a discussion of some sentences that lack the heredity property, see section 3.1.)

How does the supervaluationist semantics handle sentences (1)-(7)? (1) comes out true at $e$ in $T$ whenever each complete or semi-complete extension of $T$ contains a sea battle exactly one day after $e$. In other words, (1) is true whenever a sea battle is guaranteed true by the absolute past and present together with the laws of nature. Likewise (1) is false at $e$ in $T$ whenever no complete or semi-complete extension of $T$ contains a sea battle exactly one day after $e$-whenever the absolute past and present, together with the laws of nature, guarantee that there will be no sea battle. (1) is indeterminate whenever the absolute past, the absolute present, and the laws of nature fail to determine whether there will be a sea battle exactly one day after $e$. (2), unlike (1), comes out as either determinately true or determinately false, since all feasible timelines agree about what happened exactly one day before $e$.

(3) comes out as true when all feasible complete timelines are ones according to which there is an $e^{\prime}$ exactly one day distant from $e$ - when there is guaranteed to be a tomorrow. Likewise, (3) comes out as false when there is guaranteed not to be a tomorrow, and indeterminate when tomorrow's existence is uncertain.

(4) comes out as true in exactly the same circumstances as (3). This is somewhat surprising. (4) seems to say that the future is determined, at least as far as sea battles are concerned. After all, (4) is a disjunction, each of whose disjuncts says that the future is determined in a particular way. But (4) can be true at $e$ in $T$ even when some feasible extensions of $T$ contain a sea battle a day after $e$ and others contain no sea battle a day after $e$.

The trick is that on the supervaluationist picture, (4) can be true without either of its disjuncts being true. Consider the following example, depicted in figure 2. $e$ is the absolute present of an incomplete timeline $T$, which has two 
feasible complete extensions and no semi-complete extensions. According to one feasible history that extends $T, \mathbf{F}_{d} S$ is true at $e$; according to the other, $\mathbf{F}_{d} \neg S$ is true at $e$.

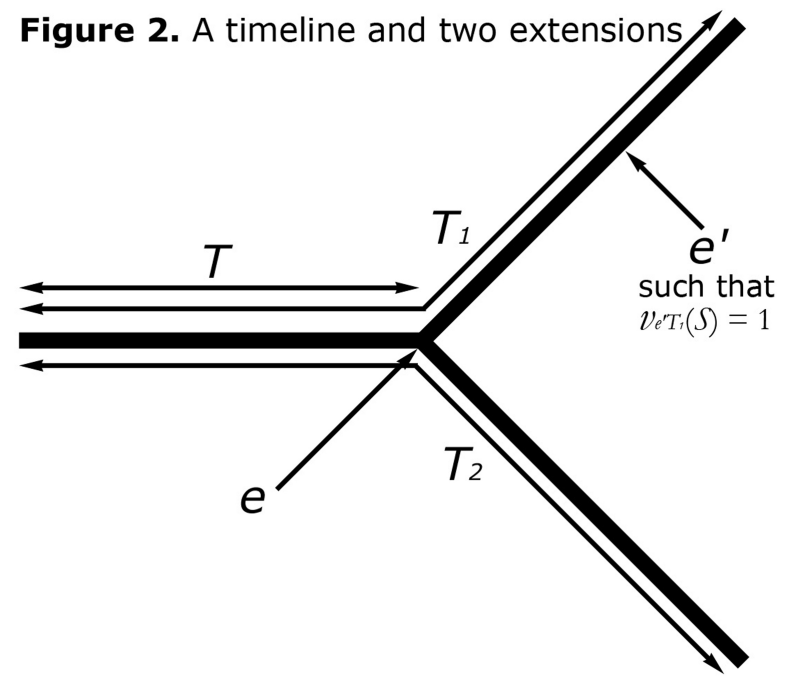

Sentence (4), the disjunction $\mathbf{F}_{d} S \vee \mathbf{F}_{d} \neg S$, is true at $e$ according to both histories that extend $T$. So by the inheritance clause, (4) is true at $e$ in $T$. But neither disjunct of (4) is true at $e$ in both histories that extend $T$-different disjuncts are true in different histories. So neither disjunct of (4) is true at $e$ in $T$.

(5) is a classical tautology - an instance of the Law of Excluded Middle - and therefore true at every time in every timeline. Like (4), (5) can be true without either of its disjuncts being true. (In fact, the reader can confirm that according to the proposed semantics, (5) will be true without either of its disjuncts being true whenever (4) is true without either of its disjuncts being true.) It seems like a point in favor of supervaluationism that it treats (5) and all other classical tautologies as valid.

(6) comes out as true at $e$ in $T$ whenever, according to all feasible timelines, there is a lottery one day after $e$, and history together with the laws of nature guarantee that the lottery will be won. (Perhaps this scenario is farfetched when it comes to real lotteries, which typically stand some chance of being called off. But we will avoid tinkering with qualifiers in order to keep the logical point as clear as possible.)

(7) comes out as true whenever (6) does. This putative equivalence is surprising, just as the putative equivalence between (3) and (4) was surprising. (7) appears to say something stronger than (6) - that the lottery is a fix. But (7) is true so long as the absolute past, the absolute present, and the laws of nature guarantee that a prize will be given out. In such circumstances, it is true according to every history that extends the actualized timeline that there is someone who will win the lottery. There need not (tenselessly) exist anyone of whom it is true that they will win the lottery - who wins the lottery on ev- 
ery history that extends the actualized timeline. Just as (4) was a disjunction that could be true without having any true disjunct, so (7) is an existentially quantified statement that can be true without having any witness.

\subsection{The Łukasiewicz Semantics}

Growing-Block theorists who find the supervaluationist treatment of (4) and (7) unsatisfactory might try a different tradeoff between (b) and (c). Instead of emphasizing the link between what is true and what is guaranteed to become true no matter how the future goes, they might emphasize the importance of preserving the meanings of certain logical connectives - in particular, the truth-functionality of of disjunction and the extensionality of existential quantification.

Supervaluationists exploited a useful insight about the future: what can be truly said of future events is roughly what is guaranteed to be true by the past, the present, and the laws of nature. But it was a mistake, opponents of supervaluationism might claim, to treat this insight as though it applied to all propositions. Propositions whose main operator is $\mathbf{F}$ are true whenever history and the laws of nature guarantee that they will become true -i.e., a proposition of the form $\mathbf{F} \phi$ is true at $e$ in $T$ whenever it is true at $e$ in all feasible extensions of $T$. But no such claim holds for propositions in general. (In particular, no such claims holds for disjunctions and existentials.) Instead, the supervaluationist's inheritance clause should be restricted to sentences whose main operators are tense operators, as follows.

- Where $\phi$ 's main operator is a tense operator,

$$
v_{e T}(\phi)=\begin{array}{ll}
1 & \text { if for every complete or semi-complete extension } T^{\prime} \text { of } T, v_{e T^{\prime}}=1 \\
0 & \text { if for every complete or semi-complete extension } T^{\prime} \text { of } T, v_{e T^{\prime}}=0
\end{array}
$$

The other connectives can be defined exactly as before, and $v_{e T}$ can be taken to be the smallest fixed point satisfying the recursive definition of the value function.

This way of defining the connections yields an extension of the logic advocated by Łukasiewicz [1970]. (Eukasiewicz outlines definitions for truthfunctional connectives and tense operators, but does not discuss quantifiers.) Unlike the supervaluationist semantics, the Eukasiewicz proposal treats the connectives as truth-functional: the truth of a disjunction (existential) will always be appropriately grounded in the truth of one or more disjuncts (witnesses).

The benefits of the Lukasiewicz system are not without their costs, however. In particular, Łukasiewicz's logic does much worse than supervaluationism on criterion (d) - it assigns truth values to far fewer sentences. As a result, it treats fewer sentences as valid - every classical tautology has untrue instances in the Eukasiewicz logic, including the law of excluded middle. ${ }^{9}$ For instance, (5) is indeterminate at $e$ in $T$ whenever some but not all semi-complete extensions of $T$

\footnotetext{
${ }^{9}$ Bourne [2006, 82-95] has proposed a modified version of Eukasiewicz's logic, which he claims allows for both non-bivalence (the idea that sentences about the future are sometimes indeterminate) and the validity of all instances of excluded middle. He does this by denying
} 
contain an $e^{\prime}$ exactly one day after $T$ at which there is a sea battle. The situation is not quite as bad as one might expect from the observation that Łukasiewicz's truth-functional logic has no theorems, however. Sentences without $\mathbf{F}$ operators or tenseless quantifiers behave classically, so that every sentence that is both valid in first-order logic and free of $\mathbf{F}$ operators and tenseless quantifiers is a theorem.

Since the Eukasiewicz semantics agrees with the supervaluationist semantics in its treatment of the $\mathbf{F}$ operator, it agrees with the supervaluationist semantics in its treatment of (1) and (2). (1) is indeterminate at $e$ in $T$ whenever T's feasible semi-complete extensions disagree about whether there is a sea battle exactly one day after $e$, while (2) is determinate at every time in every timeline.

The Eukasiewicz semantics also agrees with the supervaluationist semantics in its treatment of (3) and (6). (3) is true at $e$ in $T$ provided there will be a future -i.e., provided all of $T$ 's feasible complete or semi-complete extensions contain an $e^{\prime}$ exactly one day after $e$. And (6) is true at $e$ in $T$ whenever a prize will be given out for the lottery-i.e., whenever all of $T$ 's feasible complete or semi-complete extensions contain an $e^{\prime}$ exactly one day after $e$ at which the lottery is won. But in the Eukasiewicz semantics, unlike the supervaluationist semantics, (4) and (7) are stronger than (3) and (6). (4) is true only if one of its disjuncts is true - that is, (4) is true at $e$ in $T$ only if either all of $T$ 's feasible complete or semi-complete extensions, or none of them, contain an $e^{\prime}$ exactly one day after $e$ at which there is a sea battle. So (4) says that the past, the present, and the laws of nature settle whether there will be a sea battle tomorrow. Likewise (7) is true only if one if its instances is true - that is (7) is true at $e$ in $T$ only if the same person wins the lottery a day after $e$ in all of $T$ 's feasible extensions. So (7) says that the lottery is a fix.

\subsection{The Intuitionist Semantics}

Eukasiewicz's semantics can be strengthened by making a slight change to the definition of negation, and by adding a non-truth-functional conditional.

$$
\text { - } v_{e T}(\neg \phi)=\begin{array}{ll}
1 \quad \text { if for all complete or semi-complete extensions } T^{\prime} \text { of } T, \\
\begin{array}{l}
v_{e T^{\prime}}(\phi) \neq 1 \\
0
\end{array} \quad \text { if } v_{e T}(\phi)=1
\end{array}
$$

(a). Non-bivalence, he thinks, does not require the truth of (a), because negation should be understood as "outer" negation: $\neg \phi$ is true whenever $\phi$ is either false or indeterminate. We believe there are excellent reasons to reject Bourne's proposal. First, it is not clear how much ice non-bivalence cuts unless we reject (a) - what is the point of saying that $\phi$ is indeterminate, rather than false, if there is no sense in which $\phi$ 's negation is indeterminate rather than true? Second, Bourne's logic also requires a number of seemingly ad hoc adjustments to both the definitions of truth-functional connectives (the DeMorgan equivalences wind up invalid, and despite Bourne's non-bivalent truth assignments, every sentence or its negation is true) and the translations of English sentences into formal symbols (the apparently contingent disjunction "Either I will buy a Ducati or I will buy an MV Agusta" is translated as the tautology $(P \equiv \neg Q) \supset(P \vee Q))$. 


$$
\text { - } v_{e T}(\phi \supset \psi)=\begin{array}{ll}
1 & \text { if if for all complete or semi-complete extensions } T^{\prime} \text { of } T, \\
& v_{e T}(\phi) \neq 1 \text { or } v_{e T}(\psi)=1 \\
0 & \text { if } v_{e T}(\phi)=1 \text { and } v_{e T}(\psi) \neq 1
\end{array}
$$

Both changes can be reasonably motivated.

In the case of negation, it seems sufficient for $\neg \phi$ 's being true that $\phi$ should be guaranteed never to become true even if $\phi$ is not guaranteed to become false. Consider the example of a universe that is divided into epochs, such that the laws of nature permit it to end after any finite number of epochs, but forbid its going on for more than finitely many epochs. Consider the sentence "There will be a last time" $(\mathbf{F} \neg \mathbf{F} \top)$, as uttered sometime $e$ in the middle of the first epoch, in a timeline $T$ where $e$ is the absolute present. This sentence is not false, but it is not true, and can never become true. (After every epoch there always might be another.) So it seems reasonable to say that there won't be a last time - that $\neg \mathbf{F} \neg \mathbf{F} \top$ is true at $e$ in $T$.

The new conditional is really a strict conditional: $\phi \supset \psi$ is true at $e$ in $T$ just in case, at all feasible timelines, $\neg \phi \vee \psi$ is true at $e$. As a strict conditional, it captures the idea that the antecedent's truth necessitates the truth of the consequent, where the kind of necessity is feasibility. Notice that $\neg \phi$ is equivalent to $\phi \supset \perp$.

The result of adding the strengthened negation and conditional operators is something very close to the semantics for intuitionism proposed by Kripke [1963]. Our feasibility relation satisfies the requirements on the accessibility relation in the Kripke semantics for intuitionism - it is transitive, reflexive, antisymmetric, and (as proved in the appendix) hereditary - and our semantics defines truth for conjunctions, disjunctions, negations, conditionals, and quantified sentences in the same way as Kripke's. There are, however, a number of notable differences between our semantics and Kripke's. First, our language contains the $\mathbf{F}$ operator, which is undefined in Kripke's semantics. Second, we give falsity conditions in addition to truth conditions for our propositions. (Kripke uses the word "false" for the sentences that we are calling "untrue".)

Third, our incomplete timelines generate a slight complication. While incomplete timelines are feasible extensions of themselves, they are not complete or semi-complete feasible extensions of themselves. $T^{\prime}$ is accessible to $T$, in the sense required by Kripke's semantics, just in case $T^{\prime}$ is a complete or semicomplete extension of $T$. So even though the feasibility relation is reflexive, our analogue of Kripke's accessibility relation is not. (It is reflexive only when we restrict our attention to complete and semi-complete timelines.) This complication, however, makes little logical difference - we can simply declare each incomplete timeline to be Kripke-accessible relative to itself, without changing any logical features of the semantics.

The intuitionist semantics does better than the Lukasiewicz semantics on criterion (d). It is stronger than the Lukasiewicz semantics in two notable senses. First, at any time in any timeline, the set of true sentences according to the Eukasiewicz semantics is a subset (and sometimes a proper subset) of the set of true sentences according to the intuitionist semantics. Second, the 
set of theorems according to the Eukasiewicz semantics is a proper subset of the set of theorems according to the intuitionist semantics. (The set of inferences validated by the intuitionist semantics is neither larger nor smaller than the set of inferences validated by the Eukasiewicz semantics - they are simply incomparable.)

Our motivation for the proposed intuitionist view differs from that of traditional intuitionism, and from the intuitionism about the future (and the past) advocated by Michael Dummett [1978a,b]. Traditional intuitionists treat mathematical truth as essentially constrained by evidence, in the form of constructive proof. Dummett [1978a] extends the idea of evidential constraints on truth to empirical propositions, including propositions about times other than the present. A proposition is true for Dummett insofar as it admits of constructive proof from available evidence, and false for Dummett insofar as it admits of constructive disproof from available evidence. For Dummett, these evidential constraints on truth spring from considerations about the meanings of logical and non-logical terms - meanings are truth conditions; hence one knows the meaning of a term only insofar as one can apply it in various evidential situations.

Our approach emphasizes not evidence and meaning, but truthmaking and grounding. A sentence about the future of the form $\mathbf{F} \phi$ is true only if its truth is grounded by what exists, i.e., past and present things and the laws of nature. A sentence with any other logically complex form is true only if its truth is appropriately grounded in the truth of simpler statements-disjuncts, conjuncts, or instances. We see no reason to require that all truths about the future be knowable, or that they be grounded by knowable truths about the past and present. Our version of the intuitionist proposal requires only that they be grounded in past and present reality.

\subsection{Taking Stock}

So far, we have shown how supervaluationism, Łukasiewicz logic, and intuitionism- all ways of cashing out (a) - require different tradeoffs between (b), (c), and (d). Supervaluationists reject (b) for (c), and do better than advocates of Łukasiewicz logic and intuitionism by the lights of (d). Advocates of Łukasiewicz logic and intuitionism reject (c) for (b). Intuitionism outperforms Łukasiewicz logic by the lights of (d), but is outperformed in turn by supervaluationism.

Logicians who reject (a) must make additional tradeoffs. The choice between supervaluationism, and Łukasiewicz logic, and intuitionism has consequences for (f) - the claim that Contraposition, Reductio ad Absurdum, and Conditional Proof are valid inferences. Choosing Łukasiewicz logic means rejecting (f). Reductio ad Absurdum and Contraposition fail since classical contradictions, such as $\mathbf{F} S \wedge \neg \mathbf{F} S$, may be truth valueless rather than false, and may therefore have untrue negations. Conditional proof fails for much the same reason: $\mathbf{F} S \vDash \mathbf{F} S$, but $\mathbf{F} S \supset \mathbf{F} S$ is truth valueless whenever $\mathbf{F} S$ is.

So advocates of Łukasiewicz logic must give up (f). Intuitionists and supervaluationists have not yet been forced to give up (f), but they are not yet in 
the clear. Like most theorists who countenance truth value gaps, they must eventually choose between (f) and (e) - the claim that a language for the open future should be sufficiently powerful to include an expression for determinate truth.

Furthermore, advocates of all three logics must choose between (g), according to which truth is determinate truth, and (h), according to which truth is disquotational. The tradeoffs between (e) and (f) and (g) and (h) will be illustrated in section 3.1, where we define an operator that captures the concept of determinate truth.

Although we have more logical work to do in part 3, we are ready to conclude our discussion of the tradeoffs between the supervaluationist, Łukasiewicz, and intuitionist proposals. We believe that all three proposals are viable responses to the situation that faces the Growing-Block theorist, and that we have presented the reader with the materials necessary to make an informed choice. We now turn our attention to two logical issues that affect all three proposals equally.

\section{$3 \quad$ General Logical Issues}

The two issues to which we now turn are closely bound up with the concept of expressive power. In section 3.1, we consider how Growing-Block theorists might enrich their language to express the idea of determinate truth. Our results shed further light on the tradeoffs between (e) and (f) and (g) and (h) discussed in part 2. In section 3.2 , we consider the prospects for expressing the Growing-Block theorist's ontological commitments in a formal language.

\subsection{Determinacy}

Our metalinguistic discussion of truth at times in timelines has made extensive use of the distinction between sentences that are true (at times in timelines) and sentences that are not true (at times in timelines) - either because they are false or because they are indeterminate. How can the Growing-Block theorist express the thought that a sentence is true, rather than false or indeterminate, using an object language expression in one of the idealized languages we have developed? The usual response to this question is to define a determinacy operator. How exactly should this be done?

Thomason defines a type of determinacy operator - the inevitability operator L-as follows:

$$
\text { - } v_{e h}(\mathbf{L} \phi)=\begin{array}{ll}
1 & \text { if } v_{e h^{\prime}}(\phi)=1 \text { for every } h \text { in the model structure containing } e \\
0 & \text { else }
\end{array}
$$

How should this inevitability operator be understood in terms of truth at times in timelines?

The question is somewhat thorny. For any sentence not containing the $\mathbf{L}$ operator, we could define truth at a history, and more generally, truth at a time in a timeline. But for a sentence whose main operator is $\mathbf{L}$, we can only define 
truth at a time in a history in a model structure (and derivatively, truth at a time in a timeline in a model structure). Different choices of model structure give different truth conditions for sentences of the form $\mathbf{L} \phi$. In order to translate $\mathbf{L}$ into our formalism, then, we need a method for picking out the relevant model structure in any given situation.

One option, in keeping with the spirit of Thomason's proposal, is to index the relevant model structure (for time $e$ in timeline $T$ ) to the initial segment of $T$ containing $e$ and all the times before it. Interpreting $\mathbf{L}$ this way gives us:

$$
\text { - } v_{e T}(\mathbf{L} \phi)=\begin{array}{ll}
1 & \text { if } v_{e T^{\prime}}(\phi)=1 \\
0 & \text { else }
\end{array}
$$

where $T^{\prime}$ is the initial segment of $T$ containing only $e$ and the $e^{\prime}$ s such that $e^{\prime} R e$

This interpretation of $\mathbf{L}$ makes the model structure drop out of the semantics again - we can assign a univocal truth value at each time in each timeline to sentences of the form $\mathbf{L} \phi$.

Thomason claims that $\mathbf{L}$ has the following properties.

$$
\begin{aligned}
& \text { L1 } \phi \vDash \mathbf{L} \phi \\
& \text { L2 } \mathbf{F} \phi \vDash \mathbf{L F} \phi \\
& \text { L3 } \vdash \phi \supset \mathbf{L} \phi \\
& \text { L4 } \nvdash \models \mathbf{F} \phi \supset \mathbf{L F} \phi \\
& \text { L5 } \nvdash \models \mathbf{P F} \phi \supset \mathbf{P L F} \phi
\end{aligned}
$$

(Notice that if Thomason is right, adding $\mathbf{L}$ to the language makes it possible to generate counterexamples Conditional Proof, since $\mathbf{F} \phi \vDash \mathbf{L F} \phi$, but $\not \models \mathbf{F} \phi \supset \mathbf{L F} \phi$. Adding $\mathbf{L}$ to the language also makes it possible to generate counterexamples to Contraposition, since $\phi \vDash \mathbf{L} \phi$, but presumably $\neg \mathbf{L} \phi \not \models \phi$ )

According to our semantics for L, L4 and L5 are invalid, just as Thomason claims. Furthermore, they are invalid regardless of whether we use the supervaluationist semantics, the Łukasiewicz semantics, or the intuitionist semantics. We can produce the following countermodel for both sentences. Let $T$ be a timeline with absolute present $e$, and some $e^{*}$ such that $e^{*} R e$. And suppose $T$ has exactly two feasible complete extensions - $T_{1}$ and $T_{2}$. $T_{1}$ contains an $e^{\prime}$ such that $e R e^{\prime}$ and the atomic sentence $S$ is true at $e^{\prime} ; T_{2}$ contains no such $e^{\prime}$. Furthermore $T$ has no feasible semi-complete extensions. The example is depicted in figure 3 .

In this example, $v_{e T_{2}}(\mathbf{F} S \supset \mathbf{L F} S)=0$, so that $v_{e T}(\mathbf{F} S \supset \mathbf{L F} S) \neq 1$. Similarly, $v_{e T_{2}}(\mathbf{P F} S \supset \mathbf{P L F} S)=0$, so that $v_{e T}(\mathbf{F} S \supset \mathbf{L F} S) \neq 1$.

But according to our semantics for $\mathbf{L}$, and contrary to Thomason's claims, L1-L3 are also invalid. Once again, they are invalid regardless of whether we use the intuitionist semantics, the supervaluationist semantics, or the Eukasiewicz semantics. In the example just considered, $v_{e T_{1}}(\mathbf{F} S)=1$, while $v_{e T_{1}}(\mathbf{L F} S)=0$; 
this constitutes a counterexample to L1. Similarly, $v_{e T_{1}}(\mathbf{F} S \supset \mathbf{L F} S)=0$; this constitutes a counterexample to L2 and L3.

Although L1-L3 are not valid in general - that is, not valid $_{e T}$ - they are $\operatorname{valid}_{T}$. Thomason's model structure runs validity ${ }_{e T}$ and validity ${ }_{T}$ together. For the fragment of the language without the $\mathbf{L}$ operator, this conflation is harmless: validity ${ }_{e T}$ and validity ${ }_{T}$ coincide. But in a language containing the $\mathbf{L}$ operator, the difference matters.

Despite initial appearances to the contrary, the introduction of $\mathbf{L}$ does not not provide counterexamples to Conditional Proof or Contraposition. Enriching a language with the $\mathbf{L}$ operator preserves the heredity property (see appendix for proof). And in any supervaluationist, Łukasiewicz, or intuitionist language where all sentences satisfy the heredity property, Conditional Proof and Contraposition are valid. ${ }^{10}$

Distinguishing between validity $_{T}$ and validity ${ }_{e T}$ also reveals that $\mathbf{L}$ is a poor candidate for a truth operator. Not everything true at a time is inevitable at that time, as the invalidity of inference L1 shows. What is true full stop coincides with what is inevitable at the absolute present, but it would be a mistake to confuse truth at a time with inevitability at that time.

We can define a determinacy operator that does a better job of capturing the idea of determinate truth by making a different choice about how to pick out the relevant model structure, given a time and a timeline:

$$
\text { - } v_{e T}(\triangle \phi)=\begin{array}{ll}
1 & \text { if } v_{e T}(\phi)=1 \\
0 & \text { else }
\end{array}
$$

While $\mathbf{L} \phi$ expresses the thought that $\phi$ is determinately true at $e$ from the perspective of the initial segment of the actual timeline ending at $e, \triangle \phi$ expresses the thought that $\phi$ is true at $e$ from the perspective of the actual timeline. Determinate truth at $e$ and inevitability at $e$ correspond when $e$ is the absolute present, but come apart when $e$ is in the absolute past.

This definition of the $\triangle$ operator creates some trouble for the supervaluationist and intuitionist proposals, in which certain connectives are defined intensionally rather than truth-functionally. Sentences containing $\triangle$ violate the heredity condition - a sentence may have a truth value in one timeline without having that truth value in all of its extensions.

In supervaluationism, the failure of the heredity condition leads to inconsistent value assignments. In the example shown in figure $3, v_{e T_{1}}(\triangle \mathbf{F} S \vee \triangle \mathbf{F} \neg S)=$ $v_{e T_{2}}(\triangle \mathbf{F} S \vee \triangle \mathbf{F} \neg S)=1$ - and so by the supervaluationist inheritance clause, $v_{e T}(\triangle \mathbf{F} S \vee \triangle \mathbf{F} \neg S)$ should be 1 as well. But $v_{e T}(\triangle \mathbf{F} S)=v_{e T}(\triangle \mathbf{F} \neg S)=0$ — and so by the semantic rule for disjunction, $v_{e T}(\triangle \mathbf{F} S \vee \triangle \mathbf{F} \neg S)$ should be 0.

\footnotetext{
${ }^{10}$ Proof: Suppose $\phi \vDash \psi$, and suppose $v_{e T}(\neg \psi=1)$. By the heredity property, for every extension $T^{\prime}$ of $T, v_{e T^{\prime}}(\neg \psi)=1$. Since valuation functions are always consistent, for no extension $T^{\prime}$ of $T$ does $v_{e T^{\prime}}(\psi)=1$. Since $\phi \vDash \psi$, for no extension $T^{\prime}$ of $T$ does $v_{e T^{\prime}}(\phi)=$ 1. Whether we use the supervaluationist inheritance clause, the intuitionist clause, or the Łukasiewicz inheritance clause, this ensures that $v_{e T}(\phi)=0$.
} 
In intuitionism, the failure of the heredity condition leads to highly counterintuitive value assignments. In the example shown in figure $3, v_{e T}(\triangle \mathbf{F} S)=0$ -it is false at $e$ in $T$ that determinately, there will be a sea battle. Yet $v_{e T}(\neg \triangle \mathbf{F} S) \neq 1$. This is because at $e$ in $T$, there is some feasible timeline- namely $T_{1}$-according to which $\triangle \mathbf{F} S$ is true. Flat-footedly applying the intuitionist semantics yields counterintuitive results.

The trouble can be easily resolved by making the following adjustment to both the supervaluationist and the intuitionist semantics. To evaluate sentences containing determinacy operators at a time in $T$, first set $v_{e T^{\prime}}(\triangle \phi)$ equal to either 1 (if $v_{e T}(\phi)=1$ ) or 0 (otherwise) for every sentence $\phi$ and feasible extension $T^{\prime}$ of $T$. This ensures that the heredity condition is satisfied. Then, and only then, evaluate complex sentences in the usual way.

$\triangle$, so defined, violates the $\mathrm{T}$ schemas. The example in figure 3 ilustrates why. In this example, $v_{e T}(\mathbf{F} S) \neq 1$, so $v_{e T}(\triangle \mathbf{F} S)=v_{e T_{1}}(\triangle \mathbf{F} S)=0$. But then $v_{e T_{1}}(\phi)=1$, so that $v_{e T}(\phi \equiv \psi)=0$ according to the intuitionist semantics, and $v_{e T}(\phi \equiv \psi) \neq 1$ according to the supervaluationist semantics and Eukasiewicz semantics.

We claim that $\triangle$ behaves like an actuality operator rather than a disquotational truth operator. The values of sentences containing the $\triangle$ operator can take on different values at the same time in the same timeline, depending on which world is considered as actual. This is unsurprising, $\triangle$ denotes a type of truth that is grounded in concrete existence. One cannot tell what is true at a time simply by considering the representational features of a possible world; one needs to know which world is actualized.

No wonder, then, that there is a tradeoff between $(\mathrm{g})$, the determinacy conception of truth, and (h), the disquotational conception of truth. Determinacy, like actuality, and unlike disquotational truth, violates the $\mathrm{T}$ schema. With determinacy, as with actuality, one might be misled by the fact that the determinacy operator satisfies the T rule. For arbitrary $\phi, \phi \vDash \triangle \phi$, and $\triangle \phi \vDash \phi$. But since Conditional Proof is invalid in a language with a determinacy operator, this does not allow us to infer instances of the $\mathrm{T}$ schema. This would be just as invalid as inferring the validity of the biconditional $\ulcorner\phi \equiv$ actually $\phi\urcorner$ from the fact that $\phi$ and $\ulcorner$ actually $\phi\urcorner$ always take the same truth value at the actual world.

\subsection{Quantifier Trouble}

The eternalist quantifiers $\Sigma$ and $\Pi$ create trouble for the Growing-Block theory. Suppose that in $T$, the actualized world, $e$ is the absolute present. And let $B$ be the property of being born after $e$. Suppose the absolute past and present, together with the laws of nature, determine that someone will be born after $e$. What are we to make of the sentence $\Sigma x B x$ ?

The supervaluationist semantics counts it as determinately true that $\Sigma x B x$. But the Growing-Block theorist is committed to the claim that only past and present things exist. Things look just as bad for the intuitionist and the advocate of Łukasiewicz logic if the past and present, together with the laws of nature, 
decide who will be born after $e$. In these situations, they too will have to affirm that $\Sigma x B x$. Our semantics for the tenseless quantifiers appears to be at odds with our explicit commitments.

We reply that ontological commitment cannot be read off the tenseless existential quantifier $\Sigma$. Quantifying over everything that was, is, or will be is like quantifying over all detectives, real and fictional - a useful logical device, but not a way of stating one's ground-level commitments, since it involves quantifying over such unreal entities as Sherlock Holmes and Hercule Poirot.

How, then, should Growing-Block theorists state their ground-level ontological commitments? We suggest that a Growing-Block theorist is committed to everything that exists according to the actualized timeline. One can quantify over things that exist according to $T^{\prime}$ in the following way.

$$
\begin{gathered}
\text { - } v_{e T} \Sigma_{T^{\prime}} x(\phi x)=\begin{array}{l}
\text { if for some } e^{\prime} \text { in } T^{\prime}, \text { there is an } a \in D_{e^{\prime}} \\
\text { such that } v_{e T}(\phi x / a)=1
\end{array} \\
\text { - } v_{e T} \Pi_{T^{\prime}} x(\phi x)=\begin{array}{l}
\text { if for some } e^{\prime} \text { in } T^{\prime}, \text { there is an } a \in D_{e^{\prime}} \\
\text { such that } v_{e T}(\phi x / a)=0
\end{array}
\end{gathered}
$$

To say that $\Sigma_{T} x(\phi x)$ is to claim that some object in T's ontology is $\phi$ (at the indexical present, in the actual world).

We claim that a Growing-Block theorist is ontologically committed to everything that (by her lights) actually exists. That is, a Growing-Block theorist is ontologically committed to the existence of an object $a$ just in case she accepts that $\Sigma_{T} x(x=a)$ and $T$ is the actualized timeline.

Is there a way of introducing a formal expression for the concept of "actualized timeline"? We fear not. The formal semantics gives us a way of determining what is true at a time in a timeline, and a way determining which timeline is actualized according to each timeline (each timeline is actualized according to itself), but no way of determining which timeline is actualized full stop. Being actualized is not a matter of being actualized according to any timeline.

\section{Why The Growing Block Theory Outperforms Similar Rivals}

So far, we have developed a metaphysical and semantic framework for explicating modality in the context of the Growing-Block theory, used this framework to distinguish between true sentences about the future and false ones, developed three semantic proposals for handling the indeterminacy about the future that is likely to accompany the Growing-Block theory, and addressed questions that this indeterminacy raises about the expressive power of the languages available to Growing-Block theorists. We now turn to some of the Growing-Block theory's close relatives: Craig Bourne's ersatzer Presentism, Storrs McCall's ShrinkingTree view, and John MacFarlane's relativistic semantics for future contingents. These theories are rivals to the Growing-Block theory, since they share many of 
its appealing features. We argue that where the Growing-Block theory differs from these three proposals, it outperforms them.

\subsection{Bourne's Ersatzer Presentism}

Bourne accepts a theory of branching time much like our own, in which ersatz possible worlds consist of sequences of ersatz times. Unlike us, however, Bourne thinks that only one time-the present-is concretely instantiated. Sentences of the form $\mathbf{P} p$ are made true by the existence of ersatz times which represent $p$ as being true, and which are appropriately related to the present by the ordering of ersatz time. So if it is now true that Bill the Brontosaurus ate an oak leaf, then what makes this true is the existence of an ersatz time, appropriately related to the ersatz present, which represents Bill as eating an oak leaf.

We believe that Bourne's theory provides insufficient grounding for truths about the past. What makes that ersatz time appropriately related to the present? Why isn't the present related exclusively to ersatz times according to which Bill failed to eat oak leaves? Bourne thinks there is no issue here. "[A]ccording to ersatzer presentism," he writes,

what makes 'It was the case that $p$ ' true is an actually R-related ordered triple, whereas according to the tenseless theory, what makes it true is an actually earlier than-related concrete fact. Now to ask why these ordered triples are actually R-related is about as fair as asking why the concrete facts are actually earlier than-related in the tenseless theory, i.e., not at all-they just are. ${ }^{11}$

But this misses the point of the objection. Truth should supervene on being- on the concrete things that tenselessly exist, the properties and relations those things instantiate, and the laws of nature. According to Bourne's theory, however, there could be two possible worlds exactly alike with respect to the concrete things they contained, the properties and relations those concrete things instantiated, and the laws of nature, in which the ersatz present was nonetheless R-related to different ersatz past times. This, we think, grants the abstract realm of ersatz times far too much spooky autonomy.

The Growing-Block theory does better. Which timeline is actualized is determined by which concrete things tenselessly exist, the properties and relations those concrete things instantiate, and the laws of nature. Which complete timelines are feasible are determined by the whole of concrete reality. There are no brute facts about which ersatz times are appropriately related to the present: where concrete reality and the laws of nature are insufficient to uniquely determine the future, all candidate futures are possible.

\footnotetext{
${ }^{11}$ In the interest of notational uniformity, we have replaced Bourne's 'E-related' with our 'R-related'.
} 


\subsection{McCall's Shrinking-Tree View}

If we are concerned that Presentism has too much autonomy from concrete existence, we might consider Storrs McCall's view [McCall, 1994, 3], known as 'the Shrinking-Tree'. The Shrinking-Tree view, like the Growing-Block view, posits a moving absolute present and a concrete past. Unlike the GrowingBlock view, the Shrinking-Tree view holds that all the future possibilities are concrete, with branches ceasing to (tenselessly) exist as they cease to be feasible possibilities:

The universe, then, has in this model the shape of a tree, with a single four-dimensional trunk for the past and a densely branching set of four-dimensional manifolds for the future. Each of these manifolds in turn branches, so that the branching pattern is very complex and the number of branches very large.

McCall's tree-like structure is like our tree-like structure generated by a timeline and the set of its feasible extensions, but McCall's tree is made up of space-time, whereas the our tree is made up of ordered sets of propositions. A view where space-time has many future branches but only a single past trunk might seem a good way of capturing the intuitive view that the future is pregnant with possibility and the past is implacably fixed. There is no question, on McCall's view, of the truths not being grounded in concrete existence. McCall's view is not, then, vulnerable to the sort of objection that affects Bourne. There are some reasons for concern, however.

Tooley $[1997,239]$ argues that there are two considerations that count against McCall's view:

Broad's model of a dynamic world [the Growing-Block view] seems preferable to McCall's, for at least two reasons. First, it allows one to make sense of the idea of dynamic worlds that are deterministic, and this seems desirable, since deterministic worlds can, no less than indeterministic ones, be worlds where states of affairs come into existence. Secondly, given that a world containing no future states of affairs at all is rather more austere than one that contains states of affairs corresponding to all future possibilities, Broad's model is also to be preferred on grounds of simplicity.

McCall defines the present as the point at which branching first occurs. So, unless there is branching, there is no absolute present. This rules out the possibility of there being only one feasible future, on McCall's view, which means, as Tooley points out, the Shrinking-Tree view is incompatible with determinism. More than that, the Shrinking-Tree view cannot cope with a world which is deterministic for five minutes, since time could not pass for those five minutes, but rather would skip until the first branching-point. McCall's view rests on a strong claim, then: The laws of nature are, and always will be, indeterministic. Such assumptions should be avoided if we can do the same work without them. 
When Tooley described the Growing-Block as being more austere than the Shrinking-Tree he seems to understate the case. It is not just that McCall is committed to more than a Growing-Block theorist, but that McCall is committed to any number of as yet unborn children, who continually drop out of existence when they cease to become feasible. On both the Growing-Block and the Shrinking-Tree views, something ceasing to be feasible is a question of ontological change. On the Growing-Block something comes into existence which rules out the possibility, whereas on the Shrinking-Tree the possibility literally ceases to exist. This lends the Shrinking-Tree a destructive air. It might seem as though we are all guilty of mass-murder for wiping all the possibilities that did exist out of the universe. It is not clear that we ought to treat possible future persons as having the same moral status as present persons, nor that this is a consequence of McCall's view, but it seems odd that we should treat our prize-winning first novels, which we have not yet thought of and may never write as being just as real as the first short-stories that we wrote at school. That is the sense in which it seems the Growing-Block is more austere than the Shrinking-Tree.

You might worry that we face the same sort of objection from McCall that we put to Bourne. Do our ersatz times not have the same sort of spooky autonomy that Bourne's did? In short, they do not. Our ersatz times, unlike Bourne's are grounded in what (tenselessly) exists and the laws of nature. To explain what will be true we need no more than that what as already happened and the laws of nature. It is then left as a question for science whether the laws of nature and the history of the universe allow of a number of possible futures or not. The Growing-Block view does not need as much, ontologically, as the Shrinking-Tree, nor does it rest on tendentious assumptions about the indeterministic nature of the universe.

Our view also has explanatory advantages over the Shrinking-Tree view. The inevitability operator generates a convenient way of talking about lost possibilities, which McCall cannot easily replicate. For McCall the flow of time meant that branches of space-time dropped out of existence when they were no longer feasible. In our model, there exist not only about those possibilities which, given the state of the universe and the laws of nature, are feasible, but those which were feasible and are no longer.

Thus not only optimists and pessimists, but also historians, can be subjected to rational scrutiny through our account. Was it inevitable that the Government forces would win at Culloden? Well if the Government forces win according to all feasible extensions of the timeline the Growing-Block instantiates up to the start of the battle, then yes, it was inevitable. If there were feasible extensions of the timeline instantiated up to the start of the battle according to which the Government lose, then it was not inevitable. Unlike McCall, we need not have a further mechanism for accounting for possibility in addition to the one we use for feasible futures. We get an account of this ordinary sort of counterfactual possibility included in the package. 


\subsection{MacFarlane's Relativism}

MacFarlane's view, unlike the Shrinking-Tree view or Presentism, is concerned primarily with semantics rather than ontology. MacFarlane [2003] argues that the best way to capture the idea that some sentences about the future are truth valueless is to embrace relativism about truth. On MacFarlane's theory, the truth values of tensed sentences must be relativised not only to the time of utterance, but to the time of assessment.

MacFarlane asks us to consider a particular utterance of

(1) Exactly one day into the future, there will be a sea battle. $\left(\mathbf{F}_{d} S\right)$

We will call the utterer, as he does, Jake. Imagine Jake utters (1) on a Monday, and the world's history up to Monday together with the laws of nature fails to determine whether there will be a sea battle on Tuesday. MacFarlane thinks that on Monday, we should regard Jake's utterance as having an indeterminate truth value. MacFarlane accepts, as we do, that there can be two possible futures (one on which there is a sea-battle and one on which there isn't), and no reason to think that one of these futures is 'singled out' as the actual one. In such a case "symmetry conditions seem to rule out saying either the utterance is true or that it is false. Thus, it seems, we must count it as neither true nor false. This is the indeterminacy intuition MacFarlane [2003, 323]."

Next, imagine it is Tuesday, the day after Jake's utterance of (1) on Monday. Imagine also that on this particular Tuesday a sea battle is raging. MacFarlane argues we should be inclined to agree to the following:

Jake asserted yesterday that there would be a sea battle today

There is a sea battle today

So Jake's assertion was true.

When we take this retrospective view, we are driven to assign a determinate truth value to Jake's utterance: this is the determinacy intuition [MacFarlane, 2003, 325].

The indeterminacy intuition is in tension with the determinacy intuition. MacFarlane argues that we can accommodate both by making the truth of Jake's utterance relative to the context of assessment, so that his utterance has a different truth value depending on when you ask if it is true: it is indeterminate when assessed on Monday but true when assessed on Tuesday. The idea that Jake's utterance is true, false or indeterminate full stop, MacFarlane claims, should be rejected.

We (the authors) do not have to accept that there is no such thing as truth full stop: we already have the resources to accommodate both the determinacy intuition and the indeterminacy intuition. We needn't relativize truth to contexts of assessment; we can simply relativize truth to timelines. (Since timelines are possible worlds, this amounts to evaluating truth in different possible worlds.)

(1) is indeterminate on Monday in the relevant timeline where Monday is absolutely present - in that timeline, then, Jake's utterance of (1) is true. But 
(1) is false on Monday in the relevant timeline where Tuesday is absolutely present - in that timeline, Jake's utterance of (1) is false. The difference in truth values can be put down to a difference between possible worlds, rather than a difference between contexts. (Which world is actualized will change, of course, as time passes. This, on the Growing-Block view, is just what the passage of time is.) On Tuesday, observers can capture the sense in which Jake's utterance of (1) was indeterminate by noting that, although the proposition Jake asserted then was true $\left(\mathbf{P}_{d} \triangle \mathbf{F}_{d} S\right)$, it was not inevitably true $\left(\neg \mathbf{P}_{d} \mathbf{L} \triangle \mathbf{F}_{d} S\right)$.

There is no need to abandon truth full stop in favour of truth relative to context of assessment. What is true at a given time is dynamic, and can change as the Growing Block grows, because which world is actualized changes as the Growing Block grows. The continual change in which world is actualized is what C.D. Broad [1959, 766] described as the 'rock-bottom peculiarity' of time.

\section{Conclusion}

We have, above, considered what view we should have if we wanted to talk about the future. We have argued that someone inclined to the Growing-Block view could give a semantics that involves no commitment to anything more than what they are ontologically committed to already, but, nevertheless, can accept that there are non-trivial future truths, and that some options are feasible while others are not. Three options have been given about how this semantics might go; one supervaluationist, one Eukasiewicz, and one intuitionist. We have also considered some similar rivals, to show that our theory outperforms them.

We have not argued for the Growing-Block view itself. Equally we have not tried to deal with all of the common objections to the Growing-Block view. These topics would extend the scope of this paper beyond that which can reasonably be dealt with in a single article. We are, however, optimistic about the fortunes of what we take to be a plausible position in the philosophy of time, and believe we have shown that claiming the future does not exist, rather than rendering future possibility mysterious, allows fruitful analysis.

\section{References}

Robert Merrihew Adams. Theories of actuality. Noûs, 8(3):211-231, 1974.

Craig Bourne. A Future for Presentism. Oxford University Press, Oxford, UK, 2006.

David Braddon-Mitchell. How do we know it is now now? Analysis, 64(3): 199-203, July 2004.

C.D. Broad. Scientific Thought. Kegan Paul, London, 1923.

C.D. Broad. A reply to my critics. In P.A. Schlipp, editor, The Philosophy of C.D. Broad, pages 711-830. Tudor, New York, 1959. 
Michael Dummett. The philosophical basis of intuitionistic logic. In Truth and Other Enigmas, pages 215-247. Harvard University Press, 1978a.

Michael Dummett. The reality of the past. In Truth and Other Enigmas, pages 358-374. Harvard University Press, 1978b.

Peter Forrest. The real but dead past: a reply to Braddon-Mitchell. Analysis, 64(284):358-9, 2004.

Patrick Greenough. Indeterminate truth. Midwest Studies in Philosophy, 32: 213-241, 2008.

Saul Kripke. Semantical Analysis of Intuitionistic Logic, pages 92-130. NorthHolland, Amsterdam, 1963.

Jan Łukasiewicz. On determinism. In Ludwik Borkowski, editor, Selected Works. North-Holland, Amsterdam, 1970.

John MacFarlane. Future contingents and relative truth. The Philosophical Quarterly, 52(212):321-336, 2003.

Storrs McCall. A Model of the Universe. Clarendon Press, Oxford, UK, 1994.

Roy Sorensen. Vagueness and Contradiction. Oxford University Press, Oxford, 2001.

Richmond Thomason. Indeterminist time and truth-value gaps. Theoria, 36: 264-281, 1970.

Michael Tooley. Time, Tense, and Causation. Oxford University Press, 1997.

Achille C. Varzi. Supervaluationism and its logics. Mind, 116(463):633-676, July 2007.

\section{Appendix: Proof of the Heredity Condition}

Heredity Condition: Any sentence that receives value 0 or value 1 at $e$ in $T$ must receive the same value at $e$ in every feasible extension $T^{\prime}$ of $T$.

Base case: The heredity condition holds for atomic propositions (whose truth values at $e$ in $T$ are solely a matter of $e$ 's representational features).

Inductive Hypothesis: The heredity condition holds for propositions $\phi$ and $\psi$.

\section{Inductive Steps:}

- The heredity condition holds for $\phi \vee \psi$ and $\phi \wedge \psi$, since $\vee$ and $\wedge$ are truth functions. To inherit $v_{e T}(\phi)$ and $v_{e T}(\psi)$ is automatically to inherit $v_{e T}(\phi \vee \psi)$ and $v_{e T}(\phi \wedge \psi)$. 
- The heredity condition holds for $\neg \phi$, according to both the supervaluationist definition and the intuitionist definition of negation. On the supervaluationist definition, $\neg$ is a truth function, so that to inherit $v_{e T}(\phi)$ is automatically to inherit $v_{e T}(\neg \phi)$. On the intuitionist definition, we have two cases to consider: the case where $v_{e T}(\neg \phi)=0$, and the case where $v_{e T}(\neg \phi)=1$. If $v_{e T}(\neg \phi)=0$, then by the semantic rule for negation, $v_{e T}(\phi)=1$. By the inductive hypothesis, for every feasible extension $T^{\prime}$ of $T, v_{e T^{\prime}}(\phi)=1$. By the inutitionist semantic rule for negation, $v_{e T}(\neg \phi)=0$. If $v_{e T}(\neg \phi)=1$, then by the semantic rule for negation, at every feasible extension $T^{\prime}$ of $T, v_{e T^{\prime}}(\phi) \neq 1$. Since feasibility is transitive, for every feasible extension $T^{\prime}$ of $T$, for every feasible extension $T^{*}$ of $T^{\prime}$, $v_{e T^{*}} \neq 1$. By the semantic rule for negation $v_{e T^{\prime}}(\neg \phi)=1$.

- The heredity condition holds for $\phi \supset \psi$ in the intuitionist semantics. Whenever $v_{e T}(\phi \supset \psi)=1$, by the semantic rule for $\supset$, every feasible complete or semi-complete extension $T^{*}$ of $T$ is such that $v_{e T^{*}}(\phi) \neq 1$ or $v_{e T^{*}}(\psi)=1$. Since feasibility is transitive, for every feasible extension $T^{\prime}$ of $T$, every feasible complete extension $T^{*}$ of $T^{\prime}$ is such that either $v_{e T^{*}}(\phi) \neq 1$ or $v_{e T^{*}}(\psi)=1$. So by the semantic rule for $\supset, v_{e T^{*}}(\phi \supset \psi)=$ 1. Whenever $v_{e T}(\phi \supset \psi)=1$, by the semantic rule for $\supset, v_{e T}(\phi)=1$ and $v_{e T}(\psi)=0$. By the inductive hypothesis, where $T^{\prime}$ is any feasible extension of $T, v_{e T^{\prime}}(\phi)=1$ and $v_{e T^{\prime}}(\psi)=0$. By the semantic rule for $\supset$, $v_{e T^{\prime}}(\phi \supset \psi)=1$.

- The heredity condition holds for $\mathbf{P}(\phi)$ and $\mathbf{F}(\psi)$. This follows from the definition of an extension. By clauses (a) and (b) of the definition, whenever a timeline $T$ contains an $e$ and an $e^{\prime}$ such that $e^{\prime} R e$ and $v_{e^{\prime} T}(\phi)=1$, so does any extension - and hence, any feasible extension - of $T$. Likewise, whenever a timeline $T$ contains an $e$ and an $e^{\prime}$ such that $e R e^{\prime}$ and $v_{e^{\prime} T}(\phi)=1$, so does any (feasible) extension of $T$. Therefore, whenever $v_{e T}$ assigns value 1 to $\mathbf{P}(\phi)$ or $\mathbf{F}(\phi)$, so must $v_{e T^{\prime}}$ for any feasible extension $T^{\prime}$ of $T$. By clause (c) in the definition of an extension, whenever a timeline $T$ fails to contain an $e$ and an $e^{\prime}$ such that $e^{\prime} R e$ and $v_{e^{\prime} T}(\phi)=1$, so does any (feasible) extension of $T$. Therefore, whenever $v_{e T} \mathbf{P}(\phi)=0$, $v_{e T^{\prime}} \mathbf{P}(\phi)=0$ for any feasible extension $T^{\prime}$ of $T$.

- The heredity condition holds for $\mathbf{P}_{d}$ and $\mathbf{F}_{d}$. This follows from the definition of an extension, together with the assumption that the distance between two ersatz times depends only on the ordering, together with the representational features, of the time between them. If $T^{\prime}$ is an extension of $T$, and $T$ contains times $e$ and $e^{\prime}, T^{\prime}$ must contain $e$ and $e^{\prime}$, and will contain all the times between them in exactly the same order. Since the distance between two times supervenes on their contents and the contents and orderings of the times between them, if the distance between $e$ and $e^{\prime}$ is $d$ according to $T$, then the distance between $e$ and $e^{\prime}$ is $d$ according to $T^{\prime}$. Thus, whenever $v_{e T}\left(\mathbf{P}_{d}(\phi)\right)=1$ or $v_{e T}\left(\mathbf{P}_{d}(\phi)\right)=0$, for any extension $T^{\prime}$ 
of $T, v_{e T^{\prime}}\left(\mathbf{P}_{d}(\phi)\right)=v_{e T}\left(\mathbf{P}_{d}(\phi)\right)$, and likewise, whenever $v_{e T}\left(\mathbf{F}_{d}(\phi)\right)=1$ or $v_{e T}\left(\mathbf{F}_{d}(\phi)\right)=0$, for any extension $T^{\prime}$ of $T, v_{e T^{\prime}}\left(\mathbf{F}_{d}(\phi)\right)=v_{e T}\left(\mathbf{F}_{d}(\phi)\right)$.

- The heredity condition holds for $\Sigma x \phi$ and $\Pi x \phi$. We need consider only the case where $v_{e T}(\Sigma x \phi)=1$, and the case where $v_{e T}(\Pi x \phi)=0$. Where $v_{e T}(\Sigma x \phi x)=1$, there is an $e^{\prime} \in T$ such that for some $a \in D_{e^{\prime}}, v_{e^{\prime} T}(\phi x / a)=$ 1. Any extension $T^{\prime}$ of $T$ will also contain $e^{\prime}$, so that by the semantic rule for $\Sigma, v_{e T^{\prime}}(\Sigma x \phi x)=1$. Likewise, where $v_{e T}(\Pi x \phi x)=0$, there is an $e^{\prime} \in T$ such that for some $a \in D_{e^{\prime}}, v_{e^{\prime} T}(\phi x / a)=0$. Any extension $T^{\prime}$ of $T$ will also contain $e^{\prime}$, so that by the semantic rule for $\Pi, v_{e T^{\prime}}(\Pi x \phi x)=0$.

- The heredity condition holds for $\triangle_{T} \phi$ and $\operatorname{Tr}_{T} \phi$. By the semantic rule for $\triangle_{T}, v_{e T}\left(\triangle_{T^{*}} \phi\right)$ and $v_{e T}\left(\operatorname{Tr}_{T^{*}} \phi\right)$ are functions of $v_{e T^{*}}(\phi)$. Likewise, where $T^{\prime}$ is any feasible extension of $T, v_{e T^{\prime}}\left(\triangle_{T^{*}} \phi\right)$ and $v_{e T^{\prime}}\left(\operatorname{Tr}_{T^{*}} \phi\right)$ are the same functions of $v_{e T^{*}}(\phi)$. By the transitivity of identity, $v_{e T}\left(\triangle_{T^{*}} \phi\right)=$ $v_{e T^{\prime}}\left(\triangle_{T^{*}} \phi\right)$, and $v_{e T}\left(\boldsymbol{T r}_{T^{*}} \phi\right)=v_{e T^{\prime}}\left(\boldsymbol{T r}_{T^{*}} \phi\right)$.

- The heredity condition holds for $\mathbf{L} \phi$. By the semantic rule for $\mathbf{L}, v_{e T}(\mathbf{L} \phi)$ depends only on $v_{e T^{*}}(\phi)$, where $T^{*}$ is the initial segment of $T$ containing only $e$ and the $e^{\prime}$ such that $e R e^{\prime}$. But for any extension $T^{\prime}$ of $T, T^{*}$ is also the initial segment of $T^{\prime}$ containing only $e$ and the $e^{\prime}$ such that $e R e^{\prime}$. So $v_{e T^{\prime}} \mathbf{L} \phi=v_{e T} \mathbf{L} \phi$. 